\title{
Thermal conductivity of dilute solutions of chainlike polymers
}

\author{
C. F. Curtiss \\ Theoretical Chemistry Institute, Department of Chemistry, University of Wisconsin-Madison, Madison, \\ Wisconsin 53706 \\ R. Byron Bird \\ Chemical Engineering Department and Rheology Research Center, University of Wisconsin-Madison, \\ Madison, Wisconsin 53706
}

(Received 10 March 1997; accepted 3 June 1997)

\begin{abstract}
The Curtiss-Bird phase-space kinetic theory of polymers is used to derive an expression for the thermal conductivity of a dilute polymer solution, with the polymers represented as arbitrary bead-spring models. Then the general expression is specialized to Rouse bead-spring chains (with Hookean springs). The resulting expression contains several momentum-space averages as well as the configuration-space distribution function for the polymer chains. Use is made of the authors' previous work on the solution of the Fokker-Planck equation for arbitrary bead-spring models to evaluate the momentum-space averages. Then two special cases are considered: (a) the Hookean dumbbell model, in a fluid with velocity gradients, and (b) the Rouse chain model, with the fluid at rest. For the latter, the authors' previous study of the properties of tensor Hermite polynomials is helpful for solving the partial differential equation for the configurational distribution function for a polymer molecule in a fluid with a constant imposed temperature gradient. It is shown how the Gaussian distribution function is distorted in a nonisothermal system, but this distortion contributes only about $5 \%$ to the final value of the thermal conductivity. The results for the Rouse chain are compared with those previously obtained for several dumbbell models. () 1997 American Institute of Physics. [S0021-9606(97)50234-0]
\end{abstract}

\section{INTRODUCTION}

Studies on the thermal conductivity of dilute polymer solutions began less than a decade ago. To date the mechanical models used in the kinetic theory have been very crude, namely "elastic dumbbell" models 1 - two "beads" connected by a "spring." Three dumbbell models have been examined, corresponding to different choices for the potential energy $\phi$ of the spring:

$$
\text { Hookean spring } \quad \phi=\frac{1}{2} H Q^{2},
$$

Warner (or "FENE") spring $\phi=-\frac{1}{2} H Q_{0}^{2}\left[1-\left(Q / Q_{0}\right)^{2}\right]$,

Fraenkel spring

$$
\phi=\frac{1}{2} H\left(Q-Q_{0}\right)^{2} .
$$

Here $H$ is a spring constant, and $Q$ is the interbead distance; $Q_{0}$ in the FENE (finitely extensible nonlinear elastic) model is the length beyond which the spring cannot be stretched, and $Q_{0}$ in the Fraenkel model is the bead-bead distance when there are no forces acting on the beads. A brief summary of the conclusions from the thermal conductivity results for the three dumbbell models shown in Table I is as follows:

(a) Hookean dumbbells were first examined by van den Brule, ${ }^{2}$ and his work was extended by Curtiss and Bird ${ }^{3}$ in both of these studies, it was assumed that the velocity distribution of the beads is Maxwellian. Later, Öttinger and Petrillo ${ }^{4}$ demonstrated that this assump- tion is inadequate for calculating the third moment of the velocity distribution, which is needed for getting the heat-flux vector, and their heat-flux expression is now regarded as the best current formula for the Hookean dumbbell model. All three groups of investigators concluded that the heat flux depends on the velocity gradients in the liquid.

(b) Prior to the Öttinger-Petrillo paper, Bird and Curtiss ${ }^{5}$ obtained the thermal conductivity for a polymer solution at rest using the FENE dumbbell model and the Maxwellian velocity distribution. Subsequently, ${ }^{6}$ these results were corrected by taking into account the deviations from the Maxwellian distribution. As was to be expected, for a nonflowing liquid the thermal conductivity obtained for FENE dumbbells differs only slightly from that for Hookean dumbbells. For $Q_{0} \rightarrow \infty$ the Hookean dumbbell results are recovered.

(c) Recently, Bird, Curtiss, and Beers ${ }^{6}$ obtained the thermal conductivity for Fraenkel dumbbell solutions. For $Q_{0} \rightarrow 0$ the Hookean dumbbell results are obtained, and for $H$ large ("stiffened Fraenkel dumbbells") the model behaves something like a rigid rod, and the thermal conductivity is quite different from that of Hookean dumbbells. This development is useful in that it gives some idea as to the relation between the heat flux and the polymer stiffness.

For the dumbbell models, to get the thermal conductivity for a fluid at rest, it suffices to know the local equilibrium distribution function. That is, it is not necessary to know the 
TABLE I. Thermal conductivity contributions for several models. (Note: All entries in the table have to be multiplied by $n_{\alpha} k^{2} T / \zeta_{0}$.)

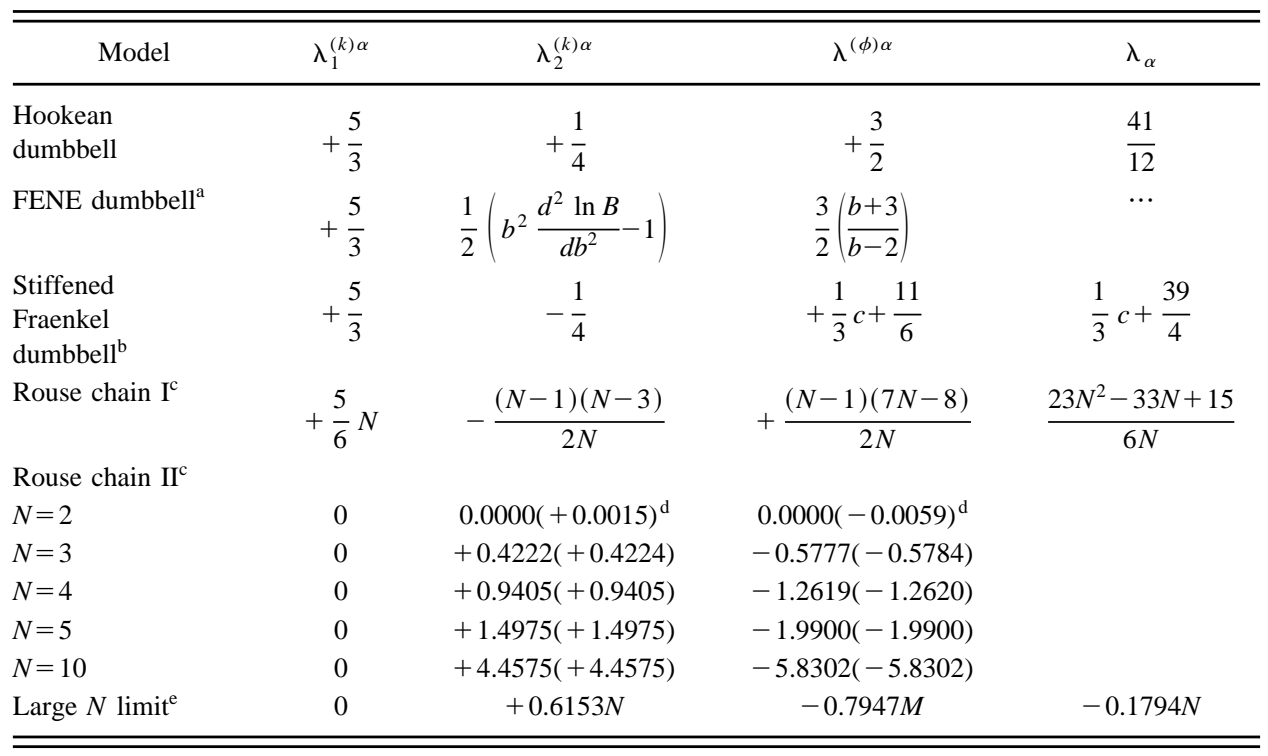

${ }^{\mathrm{a}} B$ is an abbreviation for the beta function $B(3 / 2, b / 2+1)$, where $b=H Q_{0}^{2} / k T$.

${ }^{\mathrm{b}}$ The parameter $c$ is defined as $c=H Q_{0}^{2} / 2 k T$.

" "Rouse chain I" gives the contributions from Eqs. (6.2)-(6.5), and "Rouse chain II" gives the "perturbation terms" from Eqs. (9.5) and (9.6). The contribution $\lambda_{1}^{(k) \alpha}$ consists solely of the Öttinger-Petrillo term associated with the departure of the momentum-space distribution from Maxwellian.

${ }^{\mathrm{d}}$ Quantities in parentheses are from the approximate Eqs. (9.7) and (9.8).

${ }^{\mathrm{e}} \mathrm{Q}$ untities here are from the first term of Eqs. (9.7) and (9.8).

departure of the configurational distribution function from its equilibrium shape because of the temperature gradient in the liquid. As we shall see presently, for the Rouse chain model it is necessary, even for a fluid at rest, to obtain the deviation from the equilibrium configuration distribution function when $\mathbf{a}=\nabla \ln T$ is different from zero. This proves to be the most difficult part of the development, since it involves solving a partial differential equation with $3(N-1)$ variables, where $N$ is the number of beads in the chain (usually somewhere between 30 and 300). The solution is facilitated by the use of tensor Hermite polynomials, used earlier by Curtiss and Bird for solving the one-polymer-molecule FokkerPlanck equation. ${ }^{7}$

In this publication we begin by giving the explicit expressions for the three contributions to the heat flux in dilute solutions with velocity gradients and no external forces, valid for all bead-spring models. These formal relations are obtained from the authors' previous phase-space-kinetictheory development. ${ }^{3,7}$ In these formulas there are phasespace averages, which are evaluated using our general solution to the Fokker-Planck equation for arbitrary bead-spring models. ${ }^{7}$ The expressions also contain the intramolecular potential energy and the configuration-space distribution function.

Next the general formulas are simplified for the Rouse chain model. These formulas are given for $\mathbf{a}=\nabla \ln T$ $=$ constant and for $\boldsymbol{\kappa}=(\nabla \mathbf{v})^{\dagger}=$ constant, where $(\nabla \mathbf{v})^{\dagger}$ is the transpose of the velocity-gradient tensor.
The use of these working formulas is then illustrated by obtaining the heat-flux vector for a dilute solution of Hookean dumbbells in a flow field, thereby improving on our earlier results, ${ }^{3}$ which did not include the effects of the deviations from the Maxwellian velocity distribution. The procedure used here is quite different from that used by us before, ${ }^{3}$ and is similar to that used by Öttinger and Petrillo for dumbbells. ${ }^{4}$

In a further illustration the thermal conductivity is obtained for Rouse chains in a solution at rest. First we get the various contributions to the heat flux using the equilibrium distribution function, and then we obtain the correction terms needed as a result of the distortion of the distribution function by the temperature gradients (that is, a perturbation term proportional to a).

\section{THE HEAT-FLUX VECTOR CONTRIBUTIONS IN TERMS OF MOMENTUM-SPACE AVERAGES}

The molecular theory of heat conduction in flowing polymetric liquid mixtures was developed in Secs. 8 and 16 of Ref. 3, by extending the method that Irving and Kirkwood $^{8}$ developed for monatomic liquids. It was shown that there are six mechanisms by which energy is transported in the liquid: 


$\begin{array}{ll}\mathbf{q}_{1}{ }^{(k)} & {[8.11, \text { term } 1]} \\ \mathbf{q}_{2}{ }^{(k)} & {[8.11, \text { term } 2]} \\ \mathbf{q}_{3}{ }^{(k)} & {[8.11, \text { term } 3]} \\ \mathbf{q}^{(e)} & {[8.14]} \\ \mathbf{q}^{(\phi)} & {[8.18]} \\ \mathbf{q}^{(d)} & {[8.21]}\end{array}$

kinetic transport of kinetic energy,

kinetic transport of intramolecular energy, kinetic transport of intermolecular energy, rate of doing work against external forces, rate of doing work against intramolecular forces, rate of doing work against intermolecular forces.
The rate of doing work is reckoned in a coordinate system moving with the local instantaneous mass-average velocity $\mathbf{v}(\mathbf{r}, t)$. The numbers in brackets are the equation numbers in Ref. 3; in addition, (S) shows that the contribution involves the one-molecule (singlet) distribution function, and (P) shows that the two-molecule (pair) distribution function is required. In the following development, we consider systems with no external forces, and the discussion is restricted to dilute solutions, so that intermolecular forces can be neglected. Therefore, $\mathbf{q}^{(e)}, \mathbf{q}_{3}{ }^{(k)}$, and $\mathbf{q}^{(d)}$ are not considered here.

In the following discussion, the notation of Ref. 3 is followed. For a bead $\nu$ of a molecule of species $\alpha$, the position vector, velocity, mass, and friction coefficient are given by $\mathbf{r}_{\nu}{ }^{\alpha}, \dot{\mathbf{r}}_{\nu}{ }^{\alpha}, m_{\nu}{ }^{\alpha}$, and $\zeta_{\nu}{ }^{\alpha}$. The center of mass of the molecule of species $\alpha$ is located at $\mathbf{r}_{c}{ }^{\alpha}$, and $\mathbf{R}_{\nu}{ }^{\alpha}$ is the location of bead $\nu$ with respect to the center of mass; furthermore, $\mathbf{R}_{\nu \mu}{ }^{\alpha}$ is the vector from bead $\nu$ to bead $\mu$. The vectors $\mathbf{Q}_{k}{ }^{\alpha}$ are internal coordinates representing vectors between adjacent beads arranged along an arbitrarily selected chain; in discussing the Rouse chain model these are often referred to as "connector vectors" and are chosen to be coincident with the springs. The symbols $\mathbf{r}^{\alpha}$ and $\mathbf{Q}^{\alpha}$ indicate, respectively, the set of $N_{\alpha}$ vectors $\mathbf{r}_{\nu}{ }^{\alpha}$ and the set of $N_{\alpha}-1$ vectors $\mathbf{Q}_{k}{ }^{\alpha}$. The potential energy of a molecule of species $\alpha$ is called $\phi^{\alpha}$, and the potential energy describing the interaction of two beads is called $\phi_{\nu \mu}{ }^{\alpha}$. The force acting on bead $\nu$ by bead $\mu$ is given the symbol $\mathbf{F}_{\nu \mu}{ }^{(\phi) \alpha}$, and the total force on bead $\nu$ by all other beads within the molecule is called $\mathbf{F}_{\nu}{ }^{(\phi) \alpha}$.

The formulas for the contributions of species $\alpha$ to $\mathbf{q}_{1}{ }^{(k)}$ and $\mathbf{q}_{2}{ }^{(k)}$ are taken from Table 1 Ref. 3; they are

$$
\begin{aligned}
\mathbf{q}_{1}{ }^{(k) \alpha}= & \frac{1}{2} \sum_{\nu} m_{\nu}^{\alpha} \int\left[\left[\left(\dot{\mathbf{r}}_{\nu}^{\alpha}-\mathbf{v}\right)\left(\dot{\mathbf{r}}_{\nu}^{\alpha}-\mathbf{v}\right)^{2}\right]\right]^{\alpha} \\
& \times \delta\left(\mathbf{r}_{\nu}^{\alpha}-\mathbf{r}\right) \bar{\Psi}_{\alpha}\left(\mathbf{r}^{\alpha}, t\right) d \mathbf{r}^{\alpha} \\
= & \frac{1}{2} \sum_{\nu} m_{\nu}{ }^{\alpha} \int\left[\left[\left(\dot{\mathbf{r}}_{\nu}^{\alpha}-\mathbf{v}\right)\right.\right. \\
& \left.\left.\times\left(\dot{\mathbf{r}}_{\nu}^{\alpha}-\mathbf{v}\right)^{2}\right]\right]^{\alpha} \Psi_{\alpha}\left(\mathbf{r}-\mathbf{R}_{\nu}^{\alpha}, \mathbf{Q}^{\alpha}, t\right) d \mathbf{Q}^{\alpha},
\end{aligned}
$$

$$
\begin{aligned}
\mathbf{q}_{2}{ }^{(k) \alpha} & =\frac{1}{2} \sum_{\nu \mu} \int\left[\left[\dot{\mathbf{r}}_{\nu}^{\alpha}-\mathbf{v}\right]\right]^{\alpha} \phi_{\nu \mu}^{\alpha} \delta\left(\mathbf{r}_{\nu}^{\alpha}-\mathbf{r}\right) \bar{\Psi}_{\alpha}\left(\mathbf{r}^{\alpha}, t\right) d \mathbf{r}^{\alpha} \\
& =\frac{1}{2} \sum_{\nu \mu} \int\left[\left[\dot{\mathbf{r}}_{\nu}^{\alpha}-\mathbf{v}\right]\right]^{\alpha} \phi_{\nu \mu}{ }^{\alpha} \Psi_{\alpha}\left(\mathbf{r}-\mathbf{R}_{\nu}^{\alpha}, \mathbf{Q}^{\alpha}, t\right) d \mathbf{Q}^{\alpha} .
\end{aligned}
$$

The first of these describes the transport of kinetic energy $(1 / 2) m_{\nu}{ }^{\alpha}\left(\dot{\mathbf{r}}_{\nu}{ }^{\alpha}-\mathbf{v}\right)^{2}$ by the motion of the beads of molecule $\alpha$ with respect to the mass-average velocity of the fluid $\mathbf{v}$ at position $\mathbf{r}$ and time $t$. The second describes the transport of intramolecular potential energy (energy of the springs) by the bead motion. Each of these equations is written in two forms; see Eqs. (5.7) and (5.9) in Ref. 3 for the two ways of writing statistical averages. The configuration-space distribution function is designated by $\bar{\Psi}_{\alpha}$ if the bead position vectors are used as the independent variables, and $\Psi_{\alpha}$ if center of mass location and the internal coordinates are used.

Each of the formulas above involves an integration over the phase space of the polymer molecule. The double-bracket notation indicates formally the integration over the momenta, and the integration over the configuration space is shown explicitly.

To obtain the contribution to $\mathbf{q}^{(\phi)}$, we use the results of Sec. 8.3 of Ref. 3, where it is shown how the "energy source term" $Q^{(\phi)}$ can be split up into $\left(\nabla \cdot \mathbf{q}^{(\phi)}\right)$ and $\left(\nabla \cdot\left[\pi^{(\phi)}\right.\right.$ $\cdot \mathbf{v}])$. This leads to the following expression for $\mathbf{q}^{(\phi)}$ :

$$
\begin{aligned}
\mathbf{q}^{(\phi) \alpha}= & \frac{1}{2} \sum_{\nu \mu} \iint_{0}^{1} \mathbf{R}_{\nu}^{\alpha} \mathbf{F}_{\nu \mu}^{(\phi) \alpha} \cdot\left[\left[\left(\dot{\mathbf{r}}_{\nu}{ }^{\alpha}-\mathbf{v}\right)\right.\right. \\
& \left.\left.+\left(\dot{\mathbf{r}}_{\mu}^{\alpha}-\mathbf{v}\right)\right]\right]^{\alpha} \Psi_{\alpha}\left(\mathbf{r}-\xi \mathbf{R}_{\nu}{ }^{\alpha}, \mathbf{Q}^{\alpha}, t\right) d \xi d \mathbf{Q}^{\alpha} .
\end{aligned}
$$

This contribution to the heat-flux vector involves the rate at which beads do work against the springs, in a coordinate system that is moving with the mass-average velocity $\mathbf{v}(\mathbf{r}, t)$. The double-bracket momentum-space average is to be evaluated at the point in configuration space indicated by the argument of the configuration-space distribution function. It appears necessary to perform a Taylor-series expansion of the integrand about $\mathbf{r}$, and we must keep in mind that the Taylor-series expansion does not involve the flow velocity $\mathbf{v}(\mathbf{r}, t)$. Keeping three terms in the series is sufficient for generating all the terms that are linear in the concentration and temperature gradients; throughout we systematically neglect all terms that are products of concentration and temperature gradients, as well as spatial derivatives of the gra- 
dients of concentration, temperature, and velocity. In this regard we parallel the treatment in Sec. 16.4 of Ref. 3. If we correct misprints in Eq. (16.18) of Ref. 3, the result is

$$
\begin{aligned}
\mathbf{q}^{(\phi) \alpha}= & \frac{1}{2} \sum_{\nu \mu} \int \mathbf{R}_{\nu}^{\alpha} \mathbf{F}_{\nu \mu}^{(\phi) \alpha} \cdot\left[\left[\left(\dot{\mathbf{r}}_{\nu}^{\alpha}-\mathbf{v}\right)+\left(\dot{\mathbf{r}}_{\mu}^{\alpha}-\mathbf{v}\right)\right]\right]^{\alpha} \Psi_{\alpha} d \mathbf{Q}^{\alpha} \\
& -\frac{1}{4} \nabla \cdot \sum_{\nu \mu} \int \mathbf{R}_{\nu}^{\alpha} \mathbf{R}_{\nu}^{\alpha} \mathbf{F}_{\nu \mu}^{(\phi) \alpha} \cdot\left[\left[\left(\dot{\mathbf{r}}_{\nu}^{\alpha}-\mathbf{v}\right)+\left(\dot{\mathbf{r}}_{\mu}^{\alpha}-\mathbf{v}\right)\right]\right]^{\alpha} \\
& \times \Psi_{\alpha} d \mathbf{Q}^{\alpha}+\frac{1}{12} \nabla \nabla: \sum_{\nu \mu} \int \mathbf{R}_{\nu}^{\alpha} \mathbf{R}_{\nu}^{\alpha} \mathbf{R}_{\nu}^{\alpha} \mathbf{F}_{\nu \mu}^{(\phi) \alpha} \\
& \cdot\left[\left[\left(\dot{\mathbf{r}}_{\nu}^{\alpha}-\mathbf{v}\right)+\left(\dot{\mathbf{r}}_{\mu}^{\alpha}-\mathbf{v}\right)\right]\right]^{\alpha} \Psi_{\alpha} d \mathbf{Q}^{\alpha} \\
& -\frac{1}{2} \boldsymbol{\kappa}: \sum_{\nu \mu} \int \mathbf{R}_{\nu}^{\alpha} \mathbf{F}_{\nu \mu}^{(\phi) \alpha} \mathbf{R}_{\nu}^{\alpha} \Psi_{\alpha} d \mathbf{Q}^{\alpha} \\
& +\frac{1}{3} \boldsymbol{\kappa}:\left(\nabla \cdot \sum_{\nu \mu} \int \mathbf{R}_{\nu}^{\alpha} \mathbf{R}_{\nu}^{\alpha} \mathbf{F}_{\nu \mu}^{(\phi) \alpha} \mathbf{R}_{\nu}^{\alpha} \Psi_{\alpha} d \mathbf{Q}^{\alpha}\right)
\end{aligned}
$$

The last two terms, containing the transpose of the velocity gradient $\boldsymbol{\kappa}=(\nabla \mathbf{v})^{\dagger}$, are added to compensate for the fact that the $\mathbf{v}(\mathbf{r}, t)$ in the second and third terms have been operated on by the $\nabla$-operator. In all terms the arguments of $\Psi_{\alpha}$ are $\mathbf{r}, \mathbf{Q}^{\alpha}, t$. The third term above (containing $\nabla \nabla$ ) is dropped from further consideration, inasmuch as it involves products of concentration and temperature gradients; the distribution function is linear in the concentration, and the integrals give terms which contain the temperature.

\section{HEAT-FLUX VECTOR CONTRIBUTIONS AFTER THE EVALUATION OF THE MOMENTUM- SPACE AVERAGES}

In our earlier work $^{1,3,5}$ we evaluated the double-bracket momentum-space averages in Eqs. (2.1)-(2.4) by assuming a Maxwell-Boltzmann velocity distribution for the beads. However, Öttinger and Petrillo ${ }^{4}$ have shown that, although this assumption is adequate for calculating the stress tensor (where the second moment of the distribution is involved), it is not valid for evaluating the heat-flux vector (where the third moment of the distribution arises).

Therefore, in the present treatment we do not assume "equilibration in momentum space", as we have done previously. Instead we evaluate the momentum-space averages by using our recent publication, ${ }^{7}$ in which we obtained the complete momentum-space dependence of the phase-space distribution for arbitrary bead-spring models through first order in a small expansion parameter. Specifically, we have used Eqs. (10.2), (10.4), and (10.7) of Ref. 7, which are the expressions for the first, second, and third moments of the phase-space distribution function. In the derivations of these relations it was assumed that the bead-masses are identical ( $m_{0}^{\alpha}$ ) within the molecule $\alpha$; and also that the bead friction coefficients are the same $\left(\zeta_{0}^{\alpha}\right)$ for all beads of a molecule $\alpha$ and are constants. Since we are restricting the discussion here to dilute solutions, we omit the $\mathbf{W}_{\nu 1}^{\alpha}$-term in Eq. (10.2) of Ref. 7; we further omit the "acceleration term" [the last term in Eq. (10.2) of Ref. 7], as well as the last term of Eq. (10.4) of Ref. 7, since it is of higher order.

When these expressions for the momentum-space averages are substituted into the first forms of Eqs. (2.1) and (2.2), and into Eqs. (2.3) and (2.4), we then get for any bead-spring model

$$
\begin{aligned}
\mathbf{q}_{1}^{(k) \alpha}= & -\frac{5}{6} \frac{\rho_{\alpha}}{m_{0}^{\alpha}} \frac{(k T)^{2}}{\zeta_{0}^{\alpha}} \mathbf{a}-\frac{5}{2} \frac{k T}{\zeta_{0}^{\alpha}} \nabla \cdot \pi^{(\phi) \alpha} \\
& -\frac{5}{2} \frac{k T}{\zeta_{0}^{\alpha}} \nabla \frac{\rho_{\alpha}}{m_{0}^{\alpha}} k T \\
\mathbf{q}_{2}^{(k) \alpha}= & \frac{1}{2} \frac{k T}{\zeta_{0}^{\alpha}} \nabla \cdot \boldsymbol{\pi}^{(\phi) \alpha}-\frac{1}{2} \nabla \frac{k T}{\zeta_{0}^{\alpha}} \sum_{\nu \mu} \int \phi_{\nu \mu}^{\alpha} \\
& \times \Psi_{\alpha}\left(\mathbf{r}-\mathbf{R}_{\nu}^{\alpha}, \mathbf{Q}^{\alpha}, t\right) d \mathbf{Q}^{\alpha}+\frac{1}{2 \zeta_{0}^{\alpha}} \sum_{\nu \mu} \int \phi_{\nu \mu}^{\alpha} \mathbf{F}_{\nu}^{(\phi) \alpha} \\
& \times \Psi_{\alpha}\left(\mathbf{r}-\mathbf{R}_{\nu}^{\alpha}, \mathbf{Q}^{\alpha}, t\right) d \mathbf{Q}^{\alpha},
\end{aligned}
$$

$$
\begin{aligned}
& \mathbf{q}^{(\phi) \alpha}=\frac{1}{2 \zeta_{0}^{\alpha}} \sum_{\nu \mu} \iint_{0}^{1} \mathbf{R}_{\nu}^{\alpha} \mathbf{F}_{\nu \mu}^{(\phi) \alpha} \cdot\left(\mathbf{F}_{\nu}^{(\phi) \alpha}+\mathbf{F}_{\mu}^{(\phi) \alpha}\right) \Psi_{\alpha}\left(\mathbf{r}-\xi \mathbf{R}_{\nu}^{\alpha}, \mathbf{Q}^{\alpha}, t\right) d \xi d \mathbf{Q}^{\alpha}+\frac{k T}{2 \zeta_{0}^{\alpha}} \\
& \times \sum_{\nu \mu k}\left(\bar{B}_{k \nu}+\bar{B}_{k \mu}\right) \int\left(\frac{\partial}{\partial \mathbf{Q}_{k}^{\alpha}} \cdot \mathbf{F}_{\nu \mu}^{(\phi) \alpha} \mathbf{R}_{\nu}^{\alpha}\right) \Psi_{\alpha} d \mathbf{Q}^{\alpha}+\frac{1}{2} \boldsymbol{\kappa}: \sum_{\nu \mu} \int \mathbf{R}_{\mu}^{\alpha} \mathbf{F}_{\nu \mu}^{(\phi) \alpha} \mathbf{R}_{\nu}^{\alpha} \Psi_{\alpha} d \mathbf{Q}^{\alpha}+\frac{1}{12} \boldsymbol{\kappa}: \\
& \times\left(\nabla \cdot \sum_{\nu \mu} \int \mathbf{R}_{\nu}^{\alpha}\left(\mathbf{R}_{\nu}^{\alpha}-3 \mathbf{R}_{\mu}^{\alpha}\right) \mathbf{F}_{\nu \mu}^{(\phi) \alpha} \mathbf{R}_{\nu}^{\alpha} \Psi_{\alpha} d \mathbf{Q}^{\alpha}\right)+\frac{k T}{2 \zeta_{0}^{\alpha}} \mathbf{a} \cdot \sum_{\nu \mu k} \int\left(\bar{B}_{k \nu} \mathbf{R}_{\nu}^{\alpha}+\bar{B}_{k \mu} \mathbf{R}_{\mu}^{\alpha}\right) \\
& \times\left(\frac{\partial}{\partial \mathbf{Q}_{k}^{\alpha}} \cdot \mathbf{F}_{\nu \mu}^{(\phi) \alpha} \mathbf{R}_{\nu}^{\alpha}\right) \Psi_{\alpha} d \mathbf{Q}^{\alpha}-\frac{k T}{N \zeta_{0}^{\alpha}} \mathbf{a} \cdot \sum_{\nu \mu} \int \mathbf{F}_{\nu \mu}^{(\phi) \alpha} \mathbf{R}_{\nu}^{\alpha} \Psi_{\alpha} d \mathbf{Q}^{\alpha}-\frac{k T}{4 \zeta_{0}^{\alpha}} \mathbf{a} \cdot \sum_{\nu \mu k}\left(\bar{B}_{k \nu}+\bar{B}_{k \mu}\right) \\
& \times \int\left(\frac{\partial}{\partial \mathbf{Q}_{k}^{\alpha}} \cdot \mathbf{F}_{\nu \mu}^{(\phi) \alpha} \mathbf{R}_{\nu}^{\alpha} \mathbf{R}_{\nu}^{\alpha}\right) \Psi_{\alpha} d \mathbf{Q}^{\alpha}-\frac{k T}{N \zeta_{0}^{\alpha}} \nabla \cdot \sum_{\nu \mu} \int \mathbf{F}_{\nu \mu}^{(\phi) \alpha} \mathbf{R}_{\nu}^{\alpha} \Psi_{\alpha} d \mathbf{Q}^{\alpha}-\frac{k T}{4 \zeta_{0}^{\alpha}} \nabla \cdot \sum_{\nu \mu k}\left(\bar{B}_{k \nu}+\bar{B}_{k \mu}\right) \\
& \times \int\left(\frac{\partial}{\partial \mathbf{Q}_{k}^{\alpha}} \cdot \mathbf{F}_{\nu \mu}^{(\phi) \alpha} \mathbf{R}_{\nu}^{\alpha} R_{\nu}^{\alpha}\right) \Psi_{\alpha} d \mathbf{Q}^{\alpha}
\end{aligned}
$$


Here the notation $\mathbf{a}=\nabla \ln T$ is used, and the doubly underlined term is the Öttinger-Petrillo contribution ${ }^{4}$ that appears because of the distortion of the momentum-space distribution as a result of the temperature gradient.

In obtaining the expression for $\mathbf{q}^{(\phi) \alpha}$ it has been assumed that the temperature and concentration profiles are linear functions of position and constant in time. The nonsquare matrix elements $\bar{B}_{k \nu}=\delta_{k+1, \nu}-\delta_{k \nu}$ arise when derivatives with respect to $\mathbf{r}_{\nu}^{\alpha}$ are converted to derivatives with respect to $\mathbf{Q}_{k}^{\alpha}$.

The above expressions are valid for any bead-spring model in which all beads have identical masses and friction coefficients. The intramolecular stress-tensor contribution $\pi^{(\phi) \alpha}$ appearing in Eqs. (3.1) and (3.2) is given by

$$
\pi^{(\phi) \alpha}=\sum_{\alpha \nu} \iint_{0}^{1} \mathbf{R}_{\nu}^{\alpha} \mathbf{F}_{\nu}^{(\phi) \alpha} \Psi_{\alpha}\left(\mathbf{r}-\xi \mathbf{R}_{\nu}^{\alpha}, \mathbf{Q}^{\alpha}, t\right) d \xi d \mathbf{Q}^{\alpha}
$$

This expression was derived in Sec. 7.3 of Ref. 3 .

\section{HEAT-FLUX VECTOR CONTRIBUTIONS FOR DILUTE ROUSE CHAINS}

Inasmuch as we deal with just one chemical species in this and later sections, the index $\alpha$ can be dropped from most symbols without any resulting confusion. We do, however, retain the species index $\alpha$ on the contributions to the heatflux vector and the stress tensor, on the number density and the mass concentration, and on the thermal conductivity. Furthermore, instead of using $\mathbf{Q}^{\alpha}$ for the set of all the connector vectors in the argument of the distribution function, we use $\mathbf{Q}^{N-1}$, thus displaying explicitly the number of links in the chain. Also, in integrals, instead of writing $d \mathbf{Q}^{\alpha}$, we write $d \mathbf{Q}^{N-1}$.

The Rouse chain model consists of a chain of beads and Hookean springs arranged linearly, with the beads numbered sequentially $1,2, \ldots, N$. All beads have identical masses $m_{0}$ and the same friction factors $\zeta_{0}$. The configuration of a chain can be specified by giving the bead position vectors $\mathbf{r}_{\nu}$, or alternatively the center of mass position vector $\mathbf{r}_{c}$ and the connector vectors $\mathbf{Q}_{k}=\mathbf{r}_{k+1}-\mathbf{r}_{k}$. These vectors are interrelated by

$$
\mathbf{Q}_{k}=\sum_{\nu} \bar{B}_{k \nu} \mathbf{r}_{\nu} ; \quad \mathbf{r}_{\nu}=\mathbf{r}_{c}+\sum_{k} B_{\nu k} \mathbf{Q}_{k}
$$

in which

$$
\begin{aligned}
& \bar{B}_{k \nu}=\delta_{k+1, \nu}-\delta_{k \nu}, \\
& B_{\nu k}= \begin{cases}k / N & \text { for } k<\nu, \\
(k / N)-1 & \text { for } k \geqslant \nu .\end{cases}
\end{aligned}
$$

We make full use here of the properties of the nonsquare $B$ and $\bar{B}$ matrices, which are tabulated on p. 23 and in Table 15.1-1 of Ref. 1. Unless otherwise stated, we shall use Greek indices $\nu, \mu$ (running from 1 to $N_{a}$ ) for the beads; $h, i, j, k, l$ (from 1 to $N_{\alpha}-1$ ) for the "link" or "spring"' indices; and $m, n, p, q$ (going from 1 to 3 ) for the three Cartesian indices.

The potential energy for a pair of beads joined by a Hookean spring with spring constant $H$ [needed in Eq. (3.2)] is

$$
\phi_{\nu \mu}=\frac{1}{2} H\left(\mathbf{r}_{\nu}-\mathbf{r}_{\mu}\right)^{2}\left(\delta_{\mu, \nu-1}+\delta_{\mu, \nu+1}\right),
$$

and the force on bead $\nu$ by bead $\mu$ [needed in Eq. (3.3)] is

$$
\mathbf{F}_{\nu \mu}^{(\phi)}=-\frac{\partial}{\partial \mathbf{r}_{\nu}} \phi_{\nu \mu}=-H\left(\mathbf{r}_{\nu}-\mathbf{r}_{\mu}\right)\left(\delta_{\mu, \nu-1}+\delta_{\mu, \nu+1}\right) .
$$

The total force on bead $\nu$ by the adjacent beads in the chain is given by $\mathbf{F}_{\nu}^{(\phi)}=-H \Sigma_{j} \bar{B}_{j \nu} \mathbf{Q}_{j}$.

For a dilute solution of Rouse chains, $\mathbf{q}_{1}^{(k) \alpha}$ is given by Eq. (3.1), and Eqs. (3.2) and (3.3) for $\mathbf{q}_{2}^{(k) \alpha}$ and $\mathbf{q}^{(\phi)}$ become, after inserting the expressions for the potential energies and forces (see the Appendix for some intermediate results),

$$
\begin{aligned}
\mathbf{q}_{2}^{(k) \alpha}= & \frac{1}{2} \frac{k T}{\zeta_{0}} \nabla \cdot \boldsymbol{\pi}^{(\phi) \alpha}-\frac{1}{4} \nabla \frac{H k T}{\zeta_{0}} \sum_{\nu j}\left(\delta_{\nu-1, j}+\delta_{\nu j}\right) \int\left(Q_{j}\right)^{2} \Psi\left(\mathbf{r}-\mathbf{R}_{\nu}, \mathbf{Q}^{N-1}, t\right) d \mathbf{Q}^{N-1}-\frac{H^{2}}{4 \zeta_{0}} \sum_{\nu j l} \bar{B}_{l \nu}\left(\delta_{\nu-1, j}+\delta_{\nu j}\right) \\
& \times \int\left(Q_{j}\right)^{2} \mathbf{Q}_{l} \Psi\left(\mathbf{r}-\mathbf{R}_{\nu}, \mathbf{Q}^{N-1}, t\right) d \mathbf{Q}^{N-1}, \\
\mathbf{q}^{(\phi) \alpha}= & \frac{H^{2}}{2 \zeta_{0}} \sum_{\nu j k l} X_{j k l}^{\nu} \iint_{0}^{1}\left(\mathbf{Q}_{j} \cdot \mathbf{Q}_{k}\right) \mathbf{Q}_{l} \Psi\left(\mathbf{r}-\xi \mathbf{R}_{\nu}, \mathbf{Q}^{N-1}, t\right) d \xi d \mathbf{Q}^{N-1}-\frac{(N-2) H k T}{2 N \zeta_{0}} \sum_{i} U_{i} \int \mathbf{Q}_{i} \Psi d \mathbf{Q}^{N-1} \\
& +\frac{1}{2} H \boldsymbol{\kappa}: \sum_{j k l} P_{j k l} \int \mathbf{Q}_{j} \mathbf{Q}_{k} \mathbf{Q}_{l} \Psi d \mathbf{Q}^{N-1}+\frac{1}{12} H \boldsymbol{\kappa}:\left(\nabla \cdot \sum_{j k l} N_{i j k l} \int \mathbf{Q}_{i} \mathbf{Q}_{j} \mathbf{Q}_{k} \mathbf{Q}_{l} \Psi d \mathbf{Q}^{N-1}\right) \\
& +\frac{H k T}{\zeta_{0}} \mathbf{a} \cdot \sum_{j k} S_{j k} \int \mathbf{Q}_{j} \mathbf{Q}_{k} \Psi d \mathbf{Q}^{N-1}+\frac{H k T}{\zeta_{0}} \nabla \cdot \sum_{j k} R_{j k} \int \mathbf{Q}_{j} \mathbf{Q}_{k} \Psi d \mathbf{Q}^{N-1} .
\end{aligned}
$$


The quantities $X_{j k l}^{v}, U_{i}, P_{j k l}, N_{i j k l}, S_{j k}$, and $R_{j k}$ are given in the Appendix. In the expressions for $\mathbf{q}_{1}^{(k) \alpha}$ and $\mathbf{q}_{2}^{(k) \alpha}$, the stress tensor contribution $\boldsymbol{\pi}^{(\phi) \alpha}$ for Rouse chains is

$$
\begin{aligned}
\boldsymbol{\pi}^{(\phi) \alpha}= & -H \sum_{\nu j k} B_{\nu j} \bar{B}_{k \nu} \iint_{0}^{1} \mathbf{Q}_{j} \mathbf{Q}_{k} \Psi(\mathbf{r} \\
& \left.-\xi \mathbf{R}_{\nu}, \mathbf{Q}^{N-1}, t\right) d \xi d \mathbf{Q}^{N-1} .
\end{aligned}
$$

In Eq. (4.7), the second term is zero for Hookean dumbbells, because of the factor of $N-2$; it is also zero for Rouse chains in a stationary fluid, as can be seen from the form of the configuration-space distribution function in Eqs. (6.1) and (8.23) to follow. The third term is also equal to zero for Hookean dumbbells, by virtue of the form of the configuration-space distribution function for a fluid with velocity gradients.

\section{HEAT-FLUX VECTOR EXPRESSION FOR DILUTE SYSTEMS OF HOOKEAN DUMBBELLS $(\boldsymbol{N}=2)$}

For a solution of polymers modeled as Hookean dumbbells, we are fortunate to know a great deal about the configurational distribution function. The partial differential equation for the distribution function is given in Eq. (13.17) of Ref. 3 for a general bead-spring model in a fluid with a temperature gradient. For dumbbell models, the coefficients $D_{j k l}$ are zero, so that the term containing the temperature gradient drops out, and the solution to the partial differential equation is known. The most important feature of the solution is that the connector vector $\mathbf{Q}$ appears only in a quadratic form in an exponential. This enables us to discard the third term in Eq. (4.7) for Hookean dumbbells.

For the Hookean dumbbell model (the Rouse chain of two beads with a connector vector $\mathbf{Q}), \mathbf{q}_{1}^{(k) \alpha}$ is formally given by Eq. (3.1), and $\mathbf{q}_{2}^{(k) \alpha}$ is given by the following simplification of Eq. (4.6):

$$
\begin{aligned}
\mathbf{q}_{2}^{(k) \alpha}= & \frac{1}{2} \frac{k T}{\zeta_{0}} \nabla \cdot \boldsymbol{\pi}^{(\phi) \alpha}-\frac{1}{4} \nabla \frac{H k T}{\zeta_{0}} \sum_{\nu=1}^{2} \int Q^{2} \Psi(\mathbf{r} \\
& \left.-\mathbf{R}_{\nu}, \mathbf{Q}, t\right) d \mathbf{Q}+\frac{H^{2}}{4 \zeta_{0}} \sum_{\nu=1}^{2} \int(-1)^{\nu-1} Q^{2} \mathbf{Q} \Psi(\mathbf{r} \\
& \left.-\mathbf{R}_{\nu}^{\alpha}, \mathbf{Q}, t\right) d \mathbf{Q} .
\end{aligned}
$$

In the expressions for $\mathbf{q}_{1}^{(k) \alpha}$ and $\mathbf{q}_{2}^{(k) \alpha}$ the intramolecular stress contribution $\boldsymbol{\pi}^{(\phi) \alpha}$ is now

$$
\boldsymbol{\pi}^{(\phi) \alpha}=-\frac{H}{2} \sum_{\nu} \iint_{0}^{1} \mathbf{Q Q} \Psi\left(\mathbf{r}-\xi \mathbf{R}_{\nu}, \mathbf{Q}, t\right) d \xi d \mathbf{Q},
$$

which can be obtained from Eq. (4.8).

To get the expression for $\mathbf{q}^{(\phi) \alpha}$, only the last three terms of Eq. (4.7) are nonzero for Hookean dumbbells. The constants that enter into these three terms are: $N_{1111}=-1, S_{11}$ $=-\frac{3}{2}$, and $R_{11}=+\frac{1}{2}$. Therefore, we get

$$
\begin{aligned}
\mathbf{q}^{(\phi) \alpha}= & -\frac{1}{12} H \boldsymbol{\kappa}:\left(\nabla \cdot \int \mathbf{Q Q Q Q} \Psi d \mathbf{Q}\right)-\frac{3 H k T}{2 \zeta_{0}} \mathbf{a} \\
& \cdot \int \mathbf{Q Q} \Psi d \mathbf{Q}+\frac{H k T}{2 \zeta_{0}} \nabla \cdot \int \mathbf{Q Q} \Psi d \mathbf{Q} .
\end{aligned}
$$

Because $\Psi$ for Hookean dumbbells contains $\mathbf{Q}$ only as a quadratic form in an exponential function, we need retain only one term in the Taylor-series expansion of $\boldsymbol{\pi}^{(\phi) \alpha}$, which then becomes

$$
\boldsymbol{\pi}^{(\phi) \alpha}=-n_{\alpha} k T \boldsymbol{\alpha},
$$

in which $\boldsymbol{\alpha}$ is the second-order tensor defined in Eq. (13.20) of Ref. 3; when $\mathbf{a}=\nabla \ln T$ is independent of time, this tensor is given by

$$
\begin{aligned}
\boldsymbol{\alpha}(\mathbf{r}, t)= & \frac{1}{\lambda_{H}} \int_{-\infty}^{t} e^{-\left(t-t^{\prime}\right) / \lambda_{H}} \mathbf{B}\left(t, t^{\prime}\right) d t^{\prime} \\
& -\left(\mathbf{a} \cdot \frac{1}{\lambda_{H}} \int_{-\infty}^{t} e^{-\left(t-t^{\prime}\right) / \lambda_{H}}\left(\mathbf{r}-\mathbf{r}^{\prime}\right) \mathbf{B}\left(t, t^{\prime}\right) d t^{\prime}\right),
\end{aligned}
$$

where $\lambda_{H}=\zeta_{0} / 4 H$ is the Hookean dumbbell time constant, and $\mathbf{B}$ is the Finger strain tensor (see Eq. D.3-4 of Ref. 1).

Similarly, in the second term of $\mathbf{q}_{2}^{(k) \alpha}$ in Eq. (5.1), only one term in the Taylor-series expansion is needed. However, in the third term of $\mathbf{q}_{2}^{(k) \alpha}$ we have to retain two terms in the Taylor series (the first being zero). Next, the integrals in Eqs. (5.1) and (5.3) are evaluated using Sec. 13.4 of Ref. 3. In addition, we can use Eq. (6.7) of Ref. 3 to replace $\rho_{\alpha}$ by $2 m_{0} n_{\alpha}$, since the higher-order terms may be neglected. As a result of these manipulations, the three contributions to the heat-flux vector are

$$
\begin{aligned}
\mathbf{q}_{1}^{(k) \alpha}= & -\frac{5}{3} \frac{n_{\alpha}(k T)^{2}}{\zeta_{0}} \mathbf{a}-\frac{5}{2} \frac{k T}{\zeta_{0}} \nabla \cdot\left(-n_{\alpha} k T \boldsymbol{\alpha}\right) \\
& -5 \frac{k T}{\zeta_{0}} \nabla\left(n_{\alpha} k T\right), \\
\mathbf{q}_{2}^{(k) \alpha}= & +\frac{1}{2} \frac{k T}{\zeta_{0}} \nabla \cdot\left(-n_{\alpha} k T \boldsymbol{\alpha}\right) \\
& -\frac{1}{2} \nabla\left[\frac{H k T}{\zeta_{0}}\left(\frac{n_{\alpha} k T}{H} \operatorname{tr} \boldsymbol{\alpha}\right)\right]+\frac{H^{2}}{4 \zeta_{0}} \\
& \times \nabla \cdot\left[n_{\alpha}\left(\frac{k T}{H}\right)^{2}(\boldsymbol{\alpha} \operatorname{tr} \boldsymbol{\alpha}+2 \boldsymbol{\alpha} \cdot \boldsymbol{\alpha})\right], \\
\mathbf{q}^{(\phi) \alpha}= & -\frac{1}{12} H \kappa:\left[\nabla \cdot n_{\alpha}\left(\frac{k T}{H}\right)^{2} \mathbf{I} \boldsymbol{\alpha} \boldsymbol{\alpha} \mathbf{I}\right]-\frac{3 H k T}{2 \zeta_{0}} \mathbf{a} \\
& \cdot\left(\frac{n_{\alpha} k T}{H} \boldsymbol{\alpha}\right)+\frac{H k T}{2 \zeta_{0}} \nabla \cdot\left(\frac{n_{\alpha} k T}{H} \boldsymbol{\alpha}\right),
\end{aligned}
$$

in which $\mathbf{I} \boldsymbol{\alpha} \boldsymbol{\alpha} \mathbf{I}$ is the fourth-order tensor with components $\alpha_{m n} \alpha_{p q}+\alpha_{m p} \alpha_{n q}+\alpha_{m q} \alpha_{n p}$. When these three contributions are combined, we get finally 


$$
\begin{aligned}
\mathbf{q}_{\alpha}= & -\frac{5 \frac{n_{\alpha}(k T)^{2}}{3} \frac{k T}{\zeta_{0}}}{\underline{a}-5 \frac{n_{\alpha}(k T)^{2}}{\zeta_{0}}} \nabla\left(n_{\alpha} k T\right)-4 \frac{n_{\alpha}(k T)^{2}}{\zeta_{0}}\left[\frac{5}{2} \boldsymbol{\alpha}-\frac{1}{2}(\operatorname{tr} \boldsymbol{\alpha}) \boldsymbol{\delta}+\frac{1}{2} \boldsymbol{\alpha} \cdot \boldsymbol{\alpha}\right. \\
& \left.\times[\boldsymbol{\alpha} \cdot \mathbf{a}]+\nabla \cdot \frac{n}{4}(\operatorname{tr} \boldsymbol{\alpha}) \boldsymbol{\alpha}-\frac{1}{3} \mathbf{I} \boldsymbol{\alpha} \boldsymbol{\alpha} \mathbf{I}: \lambda_{H} \boldsymbol{\kappa}\right]
\end{aligned}
$$

The expression for the heat-flux vector in Eq. (5.9) is, aside from the Ottinger-Petrillo term ${ }^{4}$ (doubly underlined), the same as that given earlier by the authors in Eq. (16.26) of Ref. 3. If we write the contribution of species $\alpha$ to the heat flux, in terms of two tensors $\boldsymbol{\beta}_{\alpha}$ and $\boldsymbol{\sigma}_{\alpha}$, as

$$
\mathbf{q}_{\alpha}=-\left[\boldsymbol{\beta}_{\alpha} \cdot \nabla T\right]-\left[\boldsymbol{\sigma}_{\alpha} \cdot \nabla n_{\alpha}\right],
$$

then for a steady shear flow of the form $v_{x}=\dot{\gamma} y$, the tensors $\boldsymbol{\beta}_{\alpha}$ and $\boldsymbol{\sigma}_{\alpha}$ are

$\boldsymbol{\beta}_{\alpha}=\frac{n_{\alpha} k T}{\zeta_{0}}\left(\begin{array}{ccc}\frac{37}{6}+\frac{31}{12} \Lambda^{2}+\frac{1}{6} \Lambda^{4} & -\frac{23}{6} \Lambda-\frac{1}{3} \Lambda^{3} & 0 \\ -\frac{1}{3} \Lambda-\frac{7}{3} \Lambda^{3} & \frac{37}{6}+2 \Lambda^{2} & 0 \\ 0 & 0 & \frac{37}{6}+\frac{5}{3} \Lambda^{2}\end{array}\right)$,

$\boldsymbol{\sigma}_{\alpha}=\frac{k^{2} T^{2}}{\zeta_{0}}\left(\begin{array}{ccc}\frac{11}{4}-\frac{15}{2} \Lambda^{2}-\Lambda^{4} & -\frac{47}{12} \Lambda-\frac{1}{6} \Lambda^{3} & 0 \\ -\frac{47}{12} \Lambda-\frac{1}{6} \Lambda^{3} & \frac{11}{4}+\Lambda^{2} & 0 \\ 0 & 0 & \frac{11}{4}+\frac{5}{6} \Lambda^{2}\end{array}\right)$

in which $\Lambda=\lambda_{H} \dot{\gamma}$ is the dimensionless shear rate. The effect of including the Öttinger-Petrillo term is to change the values of the diagonal terms in $\boldsymbol{\beta}_{\alpha}$ from $\frac{9}{2}+\cdots$ (in Ref. 3) to $\frac{37}{6}+\cdots$ in Eq. (5.11).

For systems with no velocity gradients, the tensor $\boldsymbol{\alpha}$ in Eq. (5.6) becomes the unit tensor, and the heat-flux vector is given by

$$
\mathbf{q}_{\alpha}=-\frac{n_{\alpha}(k T)^{2}}{\zeta_{0}}\left(\frac{37}{6} \mathbf{a}+\frac{11}{4} \mathbf{b}_{\alpha}\right),
$$

in which $\mathbf{b}_{\alpha}=\nabla \ln n_{\alpha}$. The thermal conductivity in a diffusing mixture is defined as the coefficient of proportionality between the heat flux and the negative of the temperature gradient, when the mass flux is set equal to zero. For any bead-spring model the mass flux is given by [cf. Eq. (15.6) of Ref. 3]:

$$
\mathbf{j}_{\alpha}=-\frac{n_{\alpha} m_{m} k T}{2 \zeta_{0}}\left(\mathbf{a}+\mathbf{b}_{\alpha}\right),
$$

where $m_{m}=2 m_{0}$ is the total mass of the dumbbell. It then follows that the contribution of species $\alpha$ to the thermal conductivity of the mixture is given by

$$
\lambda_{\alpha}=\frac{41}{12} \frac{n_{\alpha} k^{2} T}{\zeta_{0}} .
$$

In Eq. (16.33) of Ref. 3, where the thermal conductivity was obtained by assuming equilibration in momentum space (i.e., a Maxwellian velocity distribution), the numerical factor was found to be $\frac{7}{4}$. Therefore it is seen that the removal of this assumption raises the numerical coefficient appreciably.

\section{ROUSE CHAINS: THERMAL-CONDUCTIVITY CONTRIBUTIONS FROM THE EQUILIBRIUM CONFIGURATION-SPACE DISTRIBUTION FUNCTION}

In this section we begin the evaluation of the contributions to the heat-flux vector given in Eqs. (3.1), (4.6), and (4.7) using the Rouse model for a system with no velocity gradients. In this section we use the local equilibrium distribution function

$$
\begin{aligned}
\Psi_{\mathrm{eq}}=n_{\alpha} \psi_{\mathrm{eq}}= & n_{\alpha}\left(\frac{H}{2 \pi k T}\right)^{3(N-1) / 2} \\
& \times \exp \left[-(H / 2 k T) \Sigma_{k} Q_{k}^{2}\right],
\end{aligned}
$$

in which the number density of the molecules $n_{\alpha}$ and the temperature $T$ are considered to be functions of the position in the liquid. In Secs. VII to IX, we consider the deviation from this distribution function resulting from the temperature gradient in the system.

In the following, the integrals that appear can be evaluated with the help of the tabulation in Sec. 13.4 of Ref. 3. In addition, a number of summations have to be performed involving the $B_{\nu k}$ and the $\bar{B}_{k \nu}$, which appear in the quantities defined in Eqs. (A1) to (A6); for manipulating these quantities, Eqs. (2.6)-(2.12) of Ref. 3, or Sec. 11.6a and Table 15.1-1 of Ref. 1 are helpful.

The contribution $\mathbf{q}_{1}^{(k) \alpha}$ is obtained directly from Eq. (3.1) by setting $\rho_{\alpha}=N n_{\alpha} m_{0}$, where $m_{0}$ is the bead mass [cf. Eq. (6.7) of Ref. 3] in the first and third terms, and by using $\boldsymbol{\pi}^{(\phi) \alpha}=-(N-1) n_{\alpha} k T \boldsymbol{\delta}$ [cf. Eq. (14.11) of Ref. 3]. Then when the del-operations are performed we get

$$
\begin{aligned}
\mathbf{q}_{1}^{(k) \alpha}= & -\frac{5}{6} \frac{N n_{\alpha}(k T)^{2}}{\zeta_{0}}-\frac{5}{2} \frac{k T}{\zeta_{0}} \nabla \cdot\left(-(N-1) n_{\alpha} k T \boldsymbol{\delta}\right) \\
& -\frac{5}{2} \frac{k T}{\zeta_{0}} \nabla\left(N n_{\alpha} k T\right) \\
= & \frac{n_{\alpha}(k T)^{2}}{\zeta_{0}}\left[-\frac{5}{6} N \mathbf{a}+\frac{5}{2}(N-1)\left(\mathbf{a}+\mathbf{b}_{\alpha}\right)\right. \\
& \left.-\frac{5}{2} N\left(\mathbf{a}+\mathbf{b}_{\alpha}\right)\right] \\
= & \frac{n_{\alpha}(k T)^{2}}{\zeta_{0}}\left[-\left(\frac{5}{6} N+\frac{5}{2}\right) \mathbf{a}-\frac{5}{2} \mathbf{b}_{\alpha}\right],
\end{aligned}
$$

in which $\zeta_{0}$ is the friction coefficient for one bead. Here again, the doubly underlined term is the Ötinger-Petrillo term arising from the deviations of the momentum-space distribution from Maxwellian.

The contribution $\mathbf{q}_{2}^{(k) \alpha}$ in Eq. (4.6) contains three terms. The first term has been evaluated in getting Eq. (6.2). To evaluate the second term, we use the first term in the Taylorseries expansion (about position $\mathbf{r}$ ) of the distribution func- 
tion, substitute in the local equilibrium distribution function, and integrate, the integral giving $3 k T / H$. In the third term, the first term in the Taylor-series expansion of the distribution function gives zero on integration, but the second term in the expansion gives for the integral $-(3$ $\left.+2 \delta_{j l}\right) B_{\nu l} \nabla\left[n_{\alpha}(k T / H)\right]$. When the integrations are performed we get

$$
\begin{aligned}
\mathbf{q}_{2}^{(k) \alpha=} & +\frac{1}{2} \frac{k T}{\zeta_{0}} \nabla \cdot\left(-(N-1) n_{\alpha} k T \boldsymbol{\delta}\right) \\
& -\frac{1}{4} \nabla\left[\frac{k T}{\zeta_{0}} \sum_{\nu j}\left(\delta_{\nu-1, j}+\delta_{\nu, j}\right)\left(3 \frac{n_{\alpha} k T}{H}\right)\right] \\
& +\frac{H^{2}}{4 \zeta_{0}} \sum_{\nu j l} \bar{B}_{l \nu}\left(\delta_{\nu-1, j}+\delta_{\nu, j}\right) B_{\nu l} \nabla \\
& \times\left[n_{\alpha}\left(\frac{k T}{H}\right)^{2}\left(3+2 \delta_{j l}\right)\right] \\
= & \frac{n_{\alpha}(k T)^{2}}{\zeta_{0}}\left[-\frac{1}{2}(N-1)\left(\mathbf{a}+\mathbf{b}_{\alpha}\right)\right. \\
& -\frac{3}{2}(N-1)\left(2 \mathbf{a}+\mathbf{b}_{\alpha}\right) \\
& \left.+\frac{2(N-1)(4 N-3)}{4 N}\left(2 \mathbf{a}+\mathbf{b}_{\alpha}\right)\right] \\
= & \frac{n_{\alpha}(k T)^{2}}{\zeta_{0}}\left[\frac{(N-1)(N-6)}{2 N} \mathbf{a}-\frac{3(N-1)}{2 N} \mathbf{b}_{\alpha}\right] .
\end{aligned}
$$

As mentioned earlier, the second term in $\mathbf{q}^{(\phi) \alpha}$ in Eq. (4.7) vanishes for Rouse chains, and the third and fourth terms are discarded since they contain velocity gradients. The integrals in the fifth and sixth terms present no problems. In the first term of Eq. (4.7), when we expand the distribution function in a Taylor series, the first term in the series contributes nothing, since the integrand is odd in the components of the connector vectors; the second term in the Taylor expansion gives a contribution to the integral of $-\nabla \cdot \Sigma_{h} B_{\nu h}\left(3 \delta_{h l} \delta_{j k}+\delta_{h j} \delta_{k l}+\delta_{h k} \delta_{l j}\right) n_{\alpha}(k T / H)^{2} \boldsymbol{\delta}$. When the three terms arising from Eq. (4.7) are combined we get

$$
\begin{aligned}
\mathbf{q}^{(\phi) \alpha}= & -\frac{H^{2}}{2 \zeta_{0}} \sum_{\nu h j k l} X_{j k l}^{\nu} B_{\nu h}\left(3 \delta_{h l} \delta_{j k}+\delta_{h j} \delta_{k l}\right. \\
& \left.+\delta_{h k} \delta_{l j}\right) \nabla\left[n_{\alpha}\left(\frac{k T}{H}\right)^{2}\right] \\
& +\frac{H k T}{\zeta_{0}} \mathbf{a} \cdot \sum_{j} S_{j j}\left(\frac{n_{\alpha} k T}{H} \boldsymbol{\delta}\right) \\
& +\frac{H k T}{\zeta_{0}} \nabla \cdot\left[\sum_{j} R_{j j}\left(\frac{n_{\alpha} k T}{H} \boldsymbol{\delta}\right)\right] \\
= & \frac{n_{\alpha}(k T)^{2}}{\zeta_{0}}\left[-\frac{2(N-1)(N-2)}{N}\left(2 \mathbf{a}+\mathbf{b}_{\alpha}\right)\right.
\end{aligned}
$$

$$
\begin{aligned}
& \left.-\frac{3(N-1)}{2} \mathbf{a}+\frac{(N-1)}{2}\left(\mathbf{a}+\mathbf{b}_{\alpha}\right)\right] \\
= & \frac{n_{\alpha}(k T)^{2}}{\zeta_{0}} \frac{(N-1)}{2 N}\left[(-10 N+16) \mathbf{a}+(-3 N+8) \mathbf{b}_{\alpha}\right] .
\end{aligned}
$$

When the results in Eqs. (6.2)-(6.4) are combined we get for the contribution of species $\alpha$ to the heat-flux vector,

$$
\begin{aligned}
\mathbf{q}_{\alpha}= & -\frac{n_{\alpha}(k T)^{2}}{6 N \zeta_{0}}\left[2\left(16 N^{2}-21 N+15\right) \mathbf{a}\right. \\
& \left.+3\left(3 N^{2}-3 N+5\right) \mathbf{b}_{\alpha}\right] .
\end{aligned}
$$

This result simplifies to the known Hookean dumbbell results when $N$ is set equal to 2-that is, Eq. (5.9) with $\boldsymbol{\alpha}$ $=\boldsymbol{\delta}$. From Eq. (6.5) we find, by setting $\mathbf{b}_{\alpha}=-\mathbf{a}$ [from Eq. (5.14)], that the contribution of species $\alpha$ to the thermal conductivity is

$$
\lambda_{\alpha}=\left(\frac{23 N^{2}-33 N+15}{6 N}\right) \frac{n_{\alpha} k^{2} T}{\zeta_{0}} .
$$

Keep in mind that the expressions in Eqs. (6.5) and (6.6) are incomplete since they do not include the terms from the distribution function that account for the deviations from local equilibrium because of the thermal gradients. The additional terms are considered in the next several sections.

\section{ROUSE CHAINS: THE EQUATION FOR THE CONFIGURATION-SPACE DISTRIBUTION FUNCTION IN THE PRESENCE OF A TEMPERATURE GRADIENT}

In the foregoing section we used the local equilibrium distribution function $\Psi_{\mathrm{eq}}=n_{\alpha} \psi_{\mathrm{eq}}$, given in Eq. (6.1), to evaluate the heat-flux vector contributions given in Eqs. (3.1), (4.6), and (4.7). Therefore the results in Eqs. (6.2)(6.6) are incomplete, since we should use the nonequilibrium distribution function $\Psi=n_{\alpha} \psi$, which takes into account the distortion of the distribution resulting from the temperature gradient in the system.

To get this distribution function we start with the partial differential equation for $\psi$ in Eq. (13.17) of Ref. 3. This equation was derived for any bead-spring model in a fluid with velocity, concentration, and temperature gradients; it is interesting to note that the concentration gradient does not appear in this equation. In deriving this equation, the Maxwellian distribution function in momentum space (the assumption of "equilibration in momentum space") was used; however, this assumption was used only in obtaining the Brownian force term, which, according to Eq. (11.5) of Ref. 3 , involves the second moment of the momentum-space distribution. According to Eq. (10.4) of Ref. 7, the Maxwellian distribution gives a result for the second moment that differs only negligibly from that obtained from the perturbed distribution, and therefore the assumption made in getting Eq. (13.17) of Ref. 3 is inconsequential. 
When this equation for $\psi$ is written specifically for the Rouse chain model and simplified for a steady-state system in which the velocity is everywhere zero, it becomes

$$
\begin{aligned}
& \sum_{j k} A_{j k}\left(\frac{\partial}{\partial \mathbf{Q}_{j}} \cdot\left(\frac{\partial \psi}{\partial \mathbf{Q}_{k}}+\frac{H \psi}{k T} \mathbf{Q}_{k}\right)\right) \\
&+\sum_{j k l} D_{j k l}\left(\frac{\partial}{\partial \mathbf{Q}_{j}} \cdot\left(\mathbf{a} \cdot \mathbf{Q}_{l} \frac{\partial \psi}{\partial \mathbf{Q}_{k}}\right)\right)=0,
\end{aligned}
$$

in which $\mathbf{a}=\nabla \ln T$, the quantities $A_{j k}=\Sigma_{\nu} \bar{B}_{j \nu} \bar{B}_{k \nu}$ are the components of the (symmetric) Rouse matrix, and the $D_{j k l}$ are matrix components given by

$$
D_{j k l}=\sum_{\nu=1}^{N} \bar{B}_{j \nu} \bar{B}_{k \nu} B_{\nu l}, \quad \text { with } j, k, l=1,2,3, \ldots, N-1,
$$

in terms of the matrices defined in Eqs. (4.2) and (4.3). From the definition it is easily shown that $\Sigma_{j} D_{j k j}=0$ and that the $D_{j k l}$ are zero for dumbbells $(N=2)$. The term containing the $D_{j k l}$ is missing if $N=2$ or if $\mathbf{a}=0$. In either case the solution to the equation is then given by Eq. (6.1). This justifies the use of the local equilibrium distribution function for calculating the thermal conductivity for dumbbell models in systems with velocity gradients.

It is now convenient to construct two sets of tensors, $\mathbf{A}_{j k}^{[2]}$ and $\mathbf{D}_{j k l}^{[3]}$, of second and third order, respectively, with Cartesian coordinates given as follows:

$$
\left(\mathbf{A}_{j k}^{[2]}\right)_{m n}=A_{j m, k n}=A_{j k} \delta_{m n}
$$

and

$$
\left(\mathbf{D}_{j k l}^{[3]}\right)_{m n p}=D_{j m, k n, l p}=\sqrt{\frac{2 k T}{H}} D_{j k l} \delta_{m n} \hat{a}_{p} .
$$

That is, each pair of indices on $A$ and $D$ corresponds to a spring designation (running from 1 to $N-1$ ) and a Cartesiancomponent designation, with values $1,2,3$. A circumflex has been put on the components of the vector $\mathbf{a}$ in order to distinguish them from the eigenvalues of the Rouse matrix, which have been traditionally called $a_{j}$, with $j$ taking on values from 1 to $N-1$. by

We also define another set of third-order tensors ${ }^{[3]} \mathbf{D}_{j k l}$

$$
{ }^{[3]} \mathbf{D}_{j k l}=\mathbf{D}_{l k j}^{[3]}, \quad \text { or }{ }^{[3]} D_{j m, k n, l p}=D_{l p, k n, j m}^{[3]} .
$$

Because the $D_{j k l}$ are symmetric with respect to interchange of the first two indices, it follows from the definition in Eqs. (7.3) that the $\mathbf{D}_{j k l}^{[3]}$ tensors are also symmetric with respect to interchange of the first two indices. We also introduce a set of dimensionless connector vectors $\mathbf{q}_{j}=\sqrt{H / 2 k T} \mathbf{Q}_{j}$. Then Eq. (7.1) becomes

$$
\begin{aligned}
\sum_{j k} \mathbf{A}_{j k}^{[2]} & :\left(\frac{\partial}{\partial \mathbf{q}_{j}}\left(\frac{\partial \psi}{\partial \mathbf{q}_{k}}+2 \mathbf{q}_{k} \psi\right)\right) \\
& +\sum_{j k l} \frac{\partial}{\partial \mathbf{q}_{j}}\left(\left(\frac{\partial \psi}{\partial \mathbf{q}_{k}}\right) \mathbf{q}_{l}\right):^{[3]} \mathbf{D}_{l k j}=0,
\end{aligned}
$$

Note that the temperature gradient is now contained in the ${ }^{[3]} \mathbf{D}_{j k l}$ tensor.

Next, in order to make use of Appendix A of Ref. 7, we introduce vectors and tensors in a $3(N-1)$-dimensional "chain space." Specifically, q represents the set of threedimensional vectors $\mathbf{q}_{j}$, with $j=1,2,3, \ldots, N-1$. Alternatively, the vector $\mathbf{q}$ has components $q_{1 x}, q_{1 y}, q_{1 z}, q_{2 x}$, $q_{2 y}, \ldots, q_{N-1, z}$, and similar comments can be made for the differential operators and the second- and third-order tensors in Eq. (7.5). When this notation is introduced, Eq. (7.5) becomes

$$
\mathbf{A}^{[2]}: \frac{\partial}{\partial \mathbf{q}}\left(\frac{\partial \psi}{\partial \mathbf{q}}+2 \mathbf{q} \psi\right)+\frac{\partial}{\partial \mathbf{q}}\left[\left(\frac{\partial \psi}{\partial \mathbf{q}}\right) \mathbf{q}\right]:^{[3]} \mathbf{D}=0 .
$$

Equation (7.6) has to be solved to first order in a. [Note: The $\mathbf{q}$ vector here plays the same role as the $\mathbf{P}$ vector in Appendix A of Ref. 7. However, the $\mathbf{q}$ vector is in a $3(N-1)$ dimensional space, whereas the $\mathbf{P}$ vector was defined in a space of $D$ dimensions. Also note that whereas the dots in Eq. (7.5) indicate contractions on the Cartesian indices, in Eq. (7.6) they imply contractions on both the link indices and the Cartesian indices.]

Before proceeding we note that the tensors ${ }^{[3]} \mathbf{D}$ and $\mathbf{D}^{[3]}$ have the following properties:

$$
\mathbf{D}^{[3]}: \mathbf{I}^{[2]}=0, \quad \mathbf{I}^{[4]}: \mathbf{D}^{[3]}=0, \quad \mathbf{I}^{[2]}::^{[3]} \mathbf{D}=0,
$$

where the $\mathbf{I}^{[n]}$ are $n$ th-order tensors defined in Appendix A of Ref. 7 .

\section{ROUSE CHAINS: SOLUTION TO EQ. (7.6) FOR THE CONFIGURATION-SPACE DISTRIBUTION FUNCTION}

To obtain a solution to Eq. (7.6) we expand the distribution function $\psi(\mathbf{q})$ in the normalized tensor Hermite polynomials $^{9}$ presented in Appendix A of Ref. 7:

$$
\psi(\mathbf{q})=\left(\frac{H}{2 k T}\right)^{3(N-1) / 2} e^{-q^{2}} \sum_{n=0}^{\infty} \mathbf{h}^{[n] \cdot} \cdot \boldsymbol{\psi}^{[n]},
$$

in which the $\mathbf{h}^{[n]}$ are functions of $\mathbf{q}$, and ${ }^{n} \cdot$ indicates a multiple dot product. The $\psi^{[n]}$ are the expansion coefficients [cf. Eqs. (9.2) and (9.3) of Ref. 7], given by

$$
\boldsymbol{\psi}^{[n]}=\int \mathbf{h}^{[n]} \psi d \mathbf{Q}^{N-1} .
$$

We now substitute the expansion of Eq. (8.1) into the differential equation, Eq. (7.6), and then find the expansion coefficients $\boldsymbol{\psi}^{[n]}$ to first order in a. From the normalization of the distribution function and the expression for the zeroth-order Hermite function [given in Eq. (A.30) of Ref. 7], the first expansion coefficient $\boldsymbol{\psi}^{[0]}$ is known to be $\boldsymbol{\psi}^{[0]}$ $=\pi^{-3(N-1) / 4}$.

Inserting $\psi(\mathbf{q})$ from Eq. (8.1) into Eq. (7.6) and performing the indicated differentiations gives 


$$
\begin{aligned}
\mathbf{A}^{[2]} & : \sum_{n}\left(\frac{\partial}{\partial \mathbf{q}} \frac{\partial}{\partial \mathbf{q}} \mathbf{h}^{[n]}-2 \mathbf{q} \frac{\partial}{\partial \mathbf{q}} \mathbf{h}^{[n]}\right) \cdot \boldsymbol{\psi}^{[n]} \\
& +\sum_{n}\left\{\left[\frac{\partial}{\partial \mathbf{q}} \frac{\partial}{\partial \mathbf{q}} \mathbf{h}^{[n]}-2 \mathbf{q} \frac{\partial}{\partial \mathbf{q}} \mathbf{h}^{[n]}-2\left(\mathbf{q} \frac{\partial}{\partial \mathbf{q}} \mathbf{h}^{[n]}\right)\right.\right. \\
& \left.\left.+4 \mathbf{q q}^{[n]}-2 \boldsymbol{\delta}^{[2]} \mathbf{h}^{[n]}\right]^{n} \cdot \boldsymbol{\psi}^{[n]} \mathbf{q}\right\}:^{[3]} \mathbf{D} \\
& +\sum_{n}\left[\left(\frac{\partial}{\partial \mathbf{q}} \mathbf{h}^{[n]}-2 \mathbf{q h}^{[n]}\right) \cdot \boldsymbol{\psi}^{n}{ }^{[n]} \boldsymbol{\delta}^{[2]}\right]:^{[3]} \mathbf{D}=0 .
\end{aligned}
$$

The symbol " $\dagger$ ', placed to the left of a tensor indicates the transpose on the first two indices of the tensor (both link and Cartesian indices). In the first line of this equation, and also in the first two terms of the second line, we eliminate the derivatives by using Eq. (A.19) of Ref. 7; in the third line we rewrite the expression between parentheses by using Eqs. (A.12) and (A.30) of Ref. 7, and the same procedure is used to rewrite the third and fourth terms in the second line. The terms in the second line so produced are then further transformed by using Eq. (A.14) of Ref. 7.

When these operations have been performed we get the following equation for the $\psi^{[n]}$ :

$$
\begin{aligned}
\sum_{n} & {\left[\left(-2 n \boldsymbol{\delta}^{[2]} \cdot \mathbf{h}^{[n]} \cdot \mathbf{I}^{[2 n]}\right) \cdot \boldsymbol{\psi}^{[n]}\right]: \mathbf{A}^{[2]} } \\
& +\sum_{n}\left\{\left[-2 n \boldsymbol{\delta}^{[2]} \cdot \mathbf{h}^{[n]} \cdot \mathbf{I}^{[2 n]}\right.\right. \\
& +2 \sqrt{(n+1)(n+2)} \mathbf{h}^{[n+2]}+2(n+1)^{\dagger}\left(\boldsymbol{\delta}^{[2]} \mathbf{h}^{[n]}\right. \\
& n+1 \\
& \left.\left.\cdot \mathbf{I}^{[2 n+2]}\right)-2 \boldsymbol{\delta}^{[2]} \mathbf{h}^{[n]}\right] \cdot \boldsymbol{\psi}^{[n]} \mathbf{q}^{[n}::^{[3]} \mathbf{D} \\
& -\sum_{n}\left(\sqrt{2(n+1)^{\dagger}}\left(\mathbf{h}^{[n+1]}\right) \cdot \boldsymbol{\psi}^{[n]} \boldsymbol{\delta}^{[2]}\right):{ }^{[3]} \mathbf{D}=0 .
\end{aligned}
$$

Next, we multiply the equation by $e^{-q^{2} \mathbf{h}^{[n]}}$ and integrate over q. The integrals are evaluated by using Eqs. (A31) and (A32) of Ref. 7, and then $n$ is replaced by $n$. We give the results, term by term, which are

Term 1: $\quad-2 n \mathbf{I}^{[2 n]} \cdot \boldsymbol{\psi}^{[n]}: \mathbf{A}^{[2]}$

Term 2: $\quad-\sqrt{2 n}(n-1) \mathbf{I}^{[2 n]} \cdot \boldsymbol{\psi}^{[n-1]}: \mathbf{D}^{[3]}$

$$
-\sqrt{2(n+1)^{3}} \mathbf{I}^{[2 n+2]} \cdot \boldsymbol{\psi}^{[n+1]}: \mathbf{D}^{[3]},
$$

Term 3: $\quad+\sqrt{2 n(n-1)(n-2)} \mathbf{I}^{[2 n]}$

$$
\begin{aligned}
& \stackrel{n-3}{\cdot} \boldsymbol{\psi}^{[n-3]}: \mathbf{D}^{[3]}+\sqrt{2 n}(n+1) \mathbf{I}^{[2 n+2]} \\
& n-1 \\
& \quad \cdot \boldsymbol{\psi}^{[n-1]}: \mathbf{D}^{[3]},
\end{aligned}
$$

Term 4: $\quad+{\sqrt{2 n^{3}}}^{\mathbf{I}^{[2 n]}} \cdot \mathbf{I}^{[2 n]} \cdot \boldsymbol{\psi}^{[n-1]}: \mathbf{D}^{[3]}$

$$
+\sqrt{2(n+1)(n+2)} \mathbf{I}^{[2 n+2]} \cdot \mathbf{I}^{[2 n+4]^{n+1}} \cdot \boldsymbol{\psi}^{[n+1]}: \mathbf{D}^{[3]},
$$

Term 5: $\quad-\sqrt{2 n} \mathbf{I}^{[2 n]} \cdot \boldsymbol{\psi}^{[n-1]} \boldsymbol{\delta}^{[2]} \mathbf{\vdots}^{[3]}$

$$
-\sqrt{2(n+1)} \mathbf{I}^{[2 n+2]} \cdot \boldsymbol{\psi}^{[n+1]} \boldsymbol{\delta}^{[2]} \mathbf{\vdots}^{[3]},
$$

Term 6: $\quad+\sqrt{2 n} \mathbf{I}^{[2 n]} \cdot \boldsymbol{\psi}^{[n-1]} \boldsymbol{\delta}^{[2]} \mathbf{\vdots}^{[3]} \mathbf{D}$.

When these terms are inserted into Eq. (8.4), we then have the equation from which the $\boldsymbol{\psi}^{[n]}$ are to be obtained. This is done by assuming a solution of the form

$$
\boldsymbol{\psi}^{[n]}=\boldsymbol{\psi}^{[n] 0}+\boldsymbol{\psi}^{[n] 1}+\cdots,
$$

where the superscripts $0,1, \ldots$ indicate the power of $\mathbf{a}$ in the term.

At the zeroth order, namely when $\mathbf{a}=0$, terms 2 through 6 are zero because the $\mathbf{D}^{[3]}$ and ${ }^{[3]} \mathbf{D}$ tensors are linear in the components of a. Hence, we get from Eq. (8.4)

$$
2 n \mathbf{I}^{[2 n]} \cdot \boldsymbol{\psi}^{[n] 0}: \mathbf{A}^{[2]}=0 .
$$

This indicates that, at the zeroth order, all $\boldsymbol{\psi}^{[n] 0}$ are zero except for $\boldsymbol{\psi}^{[0] 0}$, which is already known to be $\pi^{-3(n-1) / 4}$ [see comments after Eq. (8.2)].

At the first order, we put the zero-order solution in all terms containing $\mathbf{a}$ in Eq. (8.4), and then we seek the solution for the first-order expansion coefficients $\boldsymbol{\psi}^{[n] 1}$ from the equation

$$
\begin{aligned}
2 n \mathbf{I}^{[2 n]} \cdot \boldsymbol{\psi}^{[n] 1}: \mathbf{A}^{[2]}= & \delta_{n 3} 2 \sqrt{3} \boldsymbol{\psi}^{[0] 0} \mathbf{I}^{[6]} \vdots \mathbf{D}^{[3]} \\
& +\delta_{n 1} 2 \sqrt{2} \boldsymbol{\psi}^{[0] 0} \mathbf{I}^{[4]} \vdots \mathbf{D}^{[3]} \\
& +\delta_{n 1} \sqrt{2} \boldsymbol{\psi}^{[0] 0} \mathbf{I}^{[2]} \mathbf{I}^{[2]} \vdots \mathbf{D}^{[3]} \\
& -\delta_{n 1} \sqrt{2} \boldsymbol{\psi}^{[0] 0} \mathbf{I}^{[2]} \boldsymbol{\delta}^{[2]} \vdots \mathbf{D}^{[3]} \\
& +\delta_{n 1} \sqrt{2} \boldsymbol{\psi}^{[0] 0} \mathbf{I}^{[2]} \boldsymbol{\delta}^{2]} \vdots^{[3]} \mathbf{D} .
\end{aligned}
$$

Term 2 in Eq. (8.5) above does not contribute; Term 3 gives the first two terms on the right side of Eq. (8.8), and Terms 4, 5 , and 6 give the last three terms. On the right side of Eq. (8.8), the third and fourth terms cancel (since $\mathbf{I}^{[2]}$ and $\boldsymbol{\delta}^{[2]}$ are identical), and the second and fifth terms are zero by the second and third relations in Eq. (7.7). This means that there is only one nonvanishing $\boldsymbol{\psi}^{[n] 1}$ in the first-order solution, namely, the tensor $\boldsymbol{\psi}^{[3] 1}$, and that it is given by Eq. (8.8) with $n$ set equal to 3

$$
6 \mathbf{I}^{[6]}: \boldsymbol{\psi}^{[3] 1}: \mathbf{A}^{[2]}=2 \sqrt{3} \boldsymbol{\psi}^{[0] 0} \mathbf{I}^{[6]}: \mathbf{D}^{[3]}
$$

or, alternatively 


$$
\mathscr{S}\left(\boldsymbol{\psi}^{[3] 1} \cdot \mathbf{A}^{[2]}\right)=\frac{\boldsymbol{\psi}^{[0] 0}}{\sqrt{3}} \mathscr{S}\left(\mathbf{D}^{[3]}\right),
$$

in which $\mathscr{S}$ is the symmetrization operator used earlier. ${ }^{7}$

From Eq. (8.10) we have to extract the components of the tensor $\boldsymbol{\psi}^{[3] 1}$. Both this tensor and the tensor $\mathbf{D}^{[3]}$ depend linearly on the vector a. We first propose a solution to Eq. (8.10) that will effectively eliminate $\mathbf{a}$ and give a relation among scalar quantities. We note from Eq. (8.2) that $\boldsymbol{\psi}^{[3]}$, and hence $\boldsymbol{\psi}^{[3] 1}$, is totally symmetric. For this reason we assert that the solution of Eq. (8.10) must have the form

$$
\boldsymbol{\psi}^{[3] 1}=\frac{\boldsymbol{\psi}^{[0] 0}}{\sqrt{3}} \mathscr{S} \mathbf{J}^{[3]},
$$

in which $\mathbf{J}^{[3]}$ is defined by

$$
\begin{aligned}
\left(\mathbf{J}_{j k l}^{[3]}\right)_{m n p}=J_{j m, k n, l p}^{[3]}= & \sqrt{\frac{2 k T}{H}} J_{j k l} \delta_{m p} \hat{a}_{n}, \\
& \text { with } J_{j k l}=J_{l k j} .
\end{aligned}
$$

Note carefully the order of the indices here, which is such that the tensor $\mathbf{J}^{[3]}$ has fore-aft symmetry with respect to both spring indices and Cartesian indices.

Substitution of $\boldsymbol{\psi}^{[3] 1}$ from Eq. (8.11) into the left side of Eq. (8.10) and taking the $j k l$-element gives

$$
\begin{gathered}
\frac{1}{3} \sum_{h}\left[\left(\mathbf{J}_{j k h}^{[3]}+\mathbf{J}_{h j k}^{[3]}+\mathbf{J}_{k h j}^{[3]}\right) \mathbf{A}_{h l}^{[2]}+\left(\mathbf{J}_{k l h}^{[3]}+\mathbf{J}_{h k l}^{[3]}+\mathbf{J}_{l h k}^{[3]}\right) \mathbf{A}_{h j}^{[2]}+\left(\mathbf{J}_{l j h}^{[3]}\right.\right. \\
\left.\left.+\mathbf{J}_{h l j}^{[3]}+\mathbf{J}_{j h l}^{[3]}\right) \mathbf{A}_{h k}^{[2]}\right]=\mathbf{D}_{j k l}^{[3]}+\mathbf{D}_{k l j}^{[3]}+\mathbf{D}_{l j k}^{[3]} .
\end{gathered}
$$

To get this result, the fore-aft symmetry of the $\mathbf{J}^{[3]}$ tensor was used. Now if terms on the left and right side are equated in the following manner:

$$
\begin{aligned}
& \frac{1}{3} \sum_{h}\left(\mathbf{J}_{k l h}^{[3]} \mathbf{A}_{h j}^{[2]}+\mathbf{J}_{h l j}^{[3]} \mathbf{A}_{h k}^{[2]}+\mathbf{J}_{k h j}^{[3]} \mathbf{A}_{h l}^{[2]}\right)=\mathbf{D}_{j k l}^{[3]}, \\
& \frac{1}{3} \sum_{h}\left(\mathbf{J}_{l j h}^{[3]} \mathbf{A}_{h k}^{[2]}+\mathbf{J}_{h j k}^{[3]} \mathbf{A}_{h l}^{[2]}+\mathbf{J}_{l h k}^{[3]} \mathbf{A}_{h j}^{[2]}\right)=\mathbf{D}_{k l j}^{[3]}, \\
& \frac{1}{3} \sum_{h}\left(\mathbf{J}_{j k h}^{[3]} \mathbf{A}_{h l}^{[2]}+\mathbf{J}_{h k l}^{[3]} \mathbf{A}_{h j}^{[2]}+\mathbf{J}_{j h l}^{[3]} \mathbf{A}_{h k}^{[2]}\right)=\mathbf{D}_{l j k}^{[3]} .
\end{aligned}
$$

It is observed that these three equations are cyclic permutations of one another. Therefore only one of them is needed, and we choose to work with Eq. (8.14). When the Cartesian components of the tensors are substituted into this equation [i.e., from Eqs. (7.3) and (8.12)], the components of the vector $\mathbf{a}$ are eliminated and a relation among scalar quantities is obtained

$$
\frac{1}{3} \sum_{h}\left(J_{k l h} A_{h j}+J_{j l h} A_{h k}+J_{k h j} A_{h l}\right)=D_{j k l} .
$$

If we use the fact that the elements of the Rouse matrix are 2 if the indices are the same and -1 if they differ by 1 , then Eq. (8.17) becomes

$$
\begin{aligned}
2 J_{j l k} & -\frac{1}{3}\left(J_{k l, j+1}+J_{j l, k+1}+J_{k, l+1, j}+J_{k, l-1, j}+J_{j l, k-1}\right. \\
& \left.+J_{k l, j-1}\right)=D_{j k l},
\end{aligned}
$$

in which it is understood that any term containing a subscript 0 or $N$ is to be discarded. Equation (8.17) [or Eq. (8.18)] is a set of simultaneous equations from which the $J_{j k l}$ can be obtained, since the $D_{j k l}$ can be constructed from Eq. (7.2) for any value of $N$.

The properties of the Rouse matrix can be used to extract the $J_{j k l}$ from Eq. (8.17). First we recall [see Eqs. (15.3-3) and (15.3-4) of Ref. 1] that there is a set of orthogonal matrices $\Omega_{j k}=\sqrt{2 / N} \sin (j k \pi / N)$ that diagonalize the Rouse matrix. That is,

$$
\sum_{j} \Omega_{i j} \Omega_{j k}=\delta_{j k}, \quad \text { and } \quad \sum_{j k} \Omega_{i j} A_{j k} \Omega_{k l}=a_{i} \delta_{i l},
$$

where the $a_{j}=4 \sin ^{2}(j \pi / 2 N)$ are the eigenvalues of the Rouse matrix. From these two relations, we get

$$
A_{j k}=\sum_{h} a_{h} \Omega_{h j} \Omega_{h k}
$$

We now substitute Eq. (8.20) into Eq. (8.17) and then multiply both sides by $\Omega_{j r} \Omega_{k s} \Omega_{l t}$ and sum on $j k l$. Then, after using Eq. (8.19), doing some rearrangement, and making use of the fore-aft symmetry of the $J_{j k l}$, we get

$$
\frac{1}{3}\left(a_{r}+a_{s}+a_{t}\right) \sum_{j k l} J_{k l j} \Omega_{j r} \Omega_{k s} \Omega_{l t}=\sum_{j k l} D_{j k l} \Omega_{j r} \Omega_{k s} \Omega_{l t} .
$$

We now divide by $\frac{1}{3}\left(a_{r}+a_{s}+a_{t}\right)$, multiply by $\Omega_{r u} \Omega_{s v} \Omega_{t w}$, and sum on rst to get

$$
\begin{aligned}
J_{v w u}= & 3 \sum_{j k l} \sum_{r s t}\left(a_{r}+a_{s}+a_{t}\right)^{-1} \\
& \times D_{j k l} \Omega_{j r} \Omega_{k s} \Omega_{l t} \Omega_{r u} \Omega_{s v} \Omega_{t w} .
\end{aligned}
$$

All indices in Eq. (8.22) run from 1 to $(N-1)$. Equations (8.11), (8.12), and (8.22) give $\boldsymbol{\psi}^{[3] 1}$.

The only nonzero elements (through first order) in Eq. (8.6) are then $\boldsymbol{\psi}^{[0]}=\boldsymbol{\psi}^{[0] 0}$ and $\boldsymbol{\psi}^{[3]}=\boldsymbol{\psi}^{[3] 1}$. This means that the solution for the configuration-space distribution function is, according to Eq. (8.1), through first order in a,

$$
\begin{aligned}
\psi(\mathbf{q})= & \left(\frac{H}{2 k T}\right)^{3(N-1) / 2} e^{-q^{2}\left[\mathbf{h}^{[0]} \boldsymbol{\psi}^{[0] 0}+\mathbf{h}^{[3]} \vdots \boldsymbol{\psi}^{[3] 1}\right]} \\
= & \psi_{\mathrm{eq}}\left[1+\frac{1}{12} \mathbf{H}^{[3]}(\mathbf{q}) \vdots \mathscr{S} \mathbf{J}^{[3]}\right] \\
= & \psi_{\mathrm{eq}}\left\{1+\frac{2}{3} \sqrt{\frac{2 k T}{H}} \sum_{i j k}\left[\left(\mathbf{q}_{j} \cdot \mathbf{q}_{k}\right) J_{j i k}\right.\right. \\
& \left.\left.-\delta_{i k}\left(J_{j j k}+\frac{3}{2} J_{j k j}\right)\right] \mathbf{q}_{i} \cdot \mathbf{a}\right\} .
\end{aligned}
$$

Thus, the correction to the local equilibrium distribution function involves just terms linear and cubic in the vectors $\mathbf{q}_{j}$. This result can be used in Eq. (9.29) of Ref. 7 to get the 
complete phase-space distribution function for a single Rouse chain in a nonflow system with a constant temperature gradient.

\section{ROUSE CHAINS: CONTRIBUTIONS TO THE THERMAL CONDUCTIVITY RESULTING FROM THE DEVIATION OF THE CONFIGURATION-SPACE DISTRIBUTION FUNCTION FROM EQUILIBRIUM}

There are only two terms in the heat-flux vector that, for a nonflow system, are affected by the second term in Eq. (8.23), namely the third term in Eq. (4.6) for $\mathbf{q}_{2}^{(k) \alpha}$ and the first term in Eq. (4.7) for $\mathbf{q}^{(\phi) \alpha}$, and in both of these, only the first term in the Taylor-series expansion is required. These terms involve contractions of integrals of the triadic product $\mathbf{Q}_{j} \mathbf{Q}_{k} \mathbf{Q}_{l}$, which can be developed as follows:

$$
\begin{aligned}
& \int \mathbf{Q}_{j} \mathbf{Q}_{k} \mathbf{Q}_{l} \psi d \mathbf{Q}^{N-1} \\
&=\left(\frac{2 k T}{H}\right)^{3 / 2} \int \mathbf{q}_{j} \mathbf{q}_{k} \mathbf{q}_{l} \psi d \mathbf{Q}^{N-1} \\
&=\left(\frac{2 k T}{H}\right)^{3 / 2} \int\left[\frac{1}{8} \mathbf{H}_{j k l}^{[3]}+\frac{3}{4} \mathscr{P}\left(\mathbf{H}^{[1]} \boldsymbol{\delta}^{[2]}\right)_{j k l}\right] \psi d \mathbf{Q}^{N-1} \\
&=\left(\frac{2 k T}{H}\right)^{3 / 2} \pi^{3(N-1) / 4} \int\left[\frac{1}{8} 2^{3 / 2} \sqrt{6} \mathbf{h}_{j k l}^{[3]}\right. \\
&\left.+\frac{1}{4} \sqrt{2} \mathscr{P}\left(\mathbf{h}^{[1]} \boldsymbol{\delta}^{2]}\right)_{j k l}\right] \psi d \mathbf{Q}^{N-1} \\
&=\left(\frac{2 k T}{H}\right)^{3 / 2} \pi^{3(N-1) / 4} \frac{1}{2} \sqrt{3} \psi_{j k l}^{[3] 1} \\
&=\left(\frac{2 k T}{H}\right)^{3 / 2} \frac{1}{6}\left(\mathbf{J}_{j k l}^{[3]}+\mathbf{J}_{k l j}^{[3]}+\mathbf{J}_{l j k}^{[3]}\right) .
\end{aligned}
$$

The manipulations performed in the first three lines are done with the help of Appendix A of Ref. 7. The term containing $\mathscr{S}\left(\mathbf{h}^{[1]} \boldsymbol{\delta}^{[2]}\right)_{j k l}$ drops out because we know that the integral of this Hermite polynomial is exactly zero [from Eqs. (8.2) and (8.23)]. The last line follows from Eq. (8.11).

In terms of Cartesian components, Eq. (9.1) is

$$
\begin{aligned}
\int Q_{j m} Q_{k n} Q_{l p} \psi d \mathbf{Q}^{N-1}= & \left(\frac{2 k T}{H}\right)^{2} \frac{1}{6}\left(J_{j k l} \delta_{m p} \hat{a}_{n}\right. \\
& \left.+J_{k l j} \delta_{n m} \hat{a}_{p}+J_{l j k} \delta_{p n} \hat{a}_{m}\right) .
\end{aligned}
$$

Since we need only the first term in the Taylor expansions of the integrands in the terms of Eqs. (4.6) and (4.7), the sums on $\nu$ can be performed, and hence we define

$$
\begin{aligned}
& \mathbf{M}_{j l}=\sum_{\nu} \bar{B}_{l \nu}\left(\delta_{\nu-1, j}+\delta_{\nu j}\right) \\
& \Lambda_{j k l}=\sum_{\nu} X_{j k l}^{\nu}=D_{j k l}+\bar{B}_{j k} B_{k+1, l}-\bar{B}_{j, k+1} B_{k l} .
\end{aligned}
$$

Equations (9.2) to (9.4) can now be used to get the "perturbation terms" in $\mathbf{q}_{2}^{(k) \alpha}$ and $\mathbf{q}^{(\phi) \alpha}$ resulting from the distortion of the configuration-space distribution function because of the temperature gradient. These are

Perturbation term in $\mathbf{q}_{2}^{(k) \alpha}=-\frac{n_{\alpha}(k T)^{2}}{6 \zeta_{0}} \sum_{j l} \mathbf{M}_{l j}\left(2 J_{j j l}\right.$

$$
\left.+3 J_{j l j}\right) \mathbf{a} \text {, }
$$

Perturbation term in $\mathbf{q}^{(\phi) \alpha}=+\frac{n_{\alpha}(k T)^{2}}{3 \zeta_{0}} \sum_{j k l} \Lambda_{j k l}\left(J_{j k l}+J_{l j k}\right.$

$$
\left.+3 J_{k l j}\right) \mathbf{a} \text {. }
$$

It is found numerically that for $N \geqslant 4$ these perturbation terms are very well described by the relations

Perturbation term in $\mathbf{q}_{2}^{(k) \alpha}=-\frac{n_{\alpha}(k T)^{2}}{N \zeta_{0}}\left(0.6153 N^{2}\right.$

$$
-1.8125 N+1.1666) \mathbf{a} \text {, }
$$

Perturbation term in $\mathbf{q}^{(\phi) \alpha}=-\frac{n_{\alpha}(k T)^{2}}{N \zeta_{0}}\left(-0.7947 N^{2}\right.$

$$
+2.2499 N-1.3329) \mathbf{a} \text {. }
$$

These terms have to be added to the "equilibrium terms" in Eqs. (6.3) and (6.4) in order to get the complete expressions for the heat-flux vector contributions for Rouse chains.

The expressions for the various contributions to the thermal conductivity for Rouse chains are shown in Table I, along with similar results for the dumbbell models. Specific results are shown for small values of $N$, as well as the limiting values for large $N$. When all the parts of the heat-flux vector are added together, from Eqs. (6.5), (9.7), and (9.8), we get finally for the polymer contribution to the thermal conductivity, for the Rouse chain model, which is good to about $0.1 \%$ down to $N=2$

$$
\lambda_{\alpha}=\left(3.6539 N^{2}-5.0626 N+2.3337\right) \frac{n_{\alpha} k^{2} T}{N \zeta_{0}} .
$$

This may be compared to the contribution of the Rouse chains to the zero-shear-rate viscosity

$$
\eta_{\alpha}=\left(\frac{N^{2}-1}{12}\right) \frac{n_{\alpha} k T \zeta_{0}}{H} .
$$

Note that $H$ does not appear in the expression for the thermal conductivity, and that the $N$ dependence is different for the two properties.

Finally, we give the intrinsic viscosity and thermal conductivity for the Rouse chain for large $N$

$$
\begin{aligned}
& {[\eta]=\lim _{\rho_{\alpha} \rightarrow 0} \frac{\eta-\eta_{s}}{\eta_{s} \rho_{\alpha}}=\frac{k T \zeta_{0}}{12 H \widetilde{N} m_{0}^{2} \eta_{s}} M_{\alpha},} \\
& {[\lambda]=\lim _{\rho_{\alpha} \rightarrow 0} \frac{\lambda-\lambda_{s}}{\lambda_{s} \rho_{\alpha}}=\frac{3.6539 k^{2} T}{m_{0} \zeta_{0} \lambda_{s}} .}
\end{aligned}
$$


These relations are obtained by letting $N=M_{\alpha} / \widetilde{N} m_{0}$ and $\rho_{\alpha}=M_{\alpha} n_{\alpha} / \widetilde{N}$, where $\widetilde{N}$ is Avogadro's number and $M_{\alpha}$ is the molecular weight of the polymer species. Equation (9.11) is Staudinger's rule, but it is known that the intrinsic viscosity is more nearly proportional to the square root of the molecular weight. We know of no data on intrinsic thermal conductivities; Eq. (9.12) suggests that this quantity should not vary very much with molecular weight.

\section{CONCLUDING COMMENTS}

Several conclusions can be drawn from this work and related studies: (a) The Öttinger-Petrillo contribution to $\lambda_{\alpha}$ for the Rouse chain model is about $23 \%$ of the total; thus the distortion of the momentum-space part of the phase-space distribution function plays an important role in the evaluation of the kinetic contribution to the heat-flux vector. (b) For large $N$ values, the contribution associated with the deviation of the configuration-space distribution function from Gaussian is about $5 \%$ of the total value. (c) The stiffened Fraenkel dumbbell model has a negative value for $\lambda_{2}^{(k) \alpha}$ and the contribution of $\lambda^{(\phi) \alpha}$ is predominant; for the other dumbbell models, each contribution is positive, and all three contributions are of importance. (d) The tensorial generalizations of the Hermite polynomials, first proposed by Grad, are useful for doing the kinetic theory of polymers in both this study and in Ref. 7. (e) It would be helpful to have experimental data for checking the validity of the theory. (f) Probably stochastic simulations will be needed for studying more realistic models and for determining the anisotropic nature of the thermal conductivity when there are velocity gradients in the fluids.

\section{ACKNOWLEDGMENT}

The authors wish to thank Professor H. C. Öttinger of ETH-Zürich for making Ref. 4 available to them when it was in the manuscript stage and for helpful interchanges of ideas during the course of this work.

\section{APPENDIX}

Here we summarize some intermediate results needed in Sec. IV in going from Eqs. (3.2) and (3.3) to Eqs. (4.6) and (4.7) for Rouse chains. First we define the following quantities that involve the Kronecker delta and the $B_{\nu k}$ and $\bar{B}_{k \nu}$ :

$$
\begin{aligned}
W_{j k}^{\nu}= & \sum_{\mu}\left(\delta_{\mu, \nu-1}+\delta_{\mu, \nu+1}\right)\left(B_{\nu j}-B_{\mu j}\right) B_{\nu k}, \\
X_{j k l}^{\nu}= & B_{\nu l}\left(\bar{B}_{j \nu} \bar{B}_{k \nu}+\bar{B}_{j, \nu-1} \delta_{\nu-1, k}-\bar{B}_{j, \nu+1} \delta_{\nu k}\right), \\
Y_{j k l}^{\nu}= & B_{\nu l}\left[2 B_{\nu j} \bar{B}_{k \nu}-\delta_{j k}\left(\delta_{\nu k}+\delta_{\nu-1, k}\right)\right], \\
Z_{j k l}^{\nu}= & B_{\nu j} \bar{B}_{k \nu} B_{\nu l}, \\
U_{k i}^{\nu \mu}= & \left(\delta_{\mu, \nu-1}+\delta_{\mu, \nu+1}\right)\left[\left(B_{\nu i}-B_{\mu i}\right) B_{\nu k}\right. \\
& \left.+3\left(B_{\nu k}-B_{\mu k}\right) B_{\nu i}\right],
\end{aligned}
$$

$$
\begin{aligned}
V_{k i j}^{\nu \mu}= & \left(\delta_{\mu, \nu-1}+\delta_{\mu, \nu+1}\right)\left[3\left(B_{\nu k}-B_{\mu k}\right) B_{\nu i} B_{\nu j}+\left(B_{\nu i}\right.\right. \\
& \left.\left.-B_{\mu i}\right) B_{\nu k} B_{\nu j}+\left(B_{\nu j}-B_{\mu j}\right) B_{\nu i} B_{\nu k}\right], \\
U_{i}= & \sum_{\nu \mu}\left(\delta_{\mu, \nu-1}+\delta_{\mu, \nu+1}\right)\left(B_{\nu i}-B_{\mu i}\right), \\
P_{j k l}= & \sum_{\nu} B_{\nu l}\left[-B_{\nu j} \bar{B}_{k \nu}+\delta_{j k}\left(\delta_{\nu k}+\delta_{\nu-1, k}\right)\right], \\
N_{i j k l}= & \sum_{\nu} B_{\nu i} B_{\nu l}\left[2 B_{\nu j} \bar{B}_{k \nu}-3 \delta_{j k}\left(\delta_{\nu k}+\delta_{\nu-1}, k\right)\right], \\
S_{j k}= & \sum_{\nu \mu}\left(\delta_{\mu, \nu-1}+\delta_{\mu, \nu+1}\right)\left(-\frac{3 N-1}{2 N} B_{\nu j} B_{\nu k}\right. \\
& \left.+\frac{1}{4} B_{\nu j} B_{\mu k}+\frac{5}{4} B_{\mu j} B_{\nu k}-\frac{1}{2 N} B_{\mu j} B_{\mu k}\right), \\
R_{j k}= & \sum_{\nu \mu}\left(\delta_{\mu, \nu-1}+\delta_{\mu, \nu+1}\right)\left(\frac{1}{2} B_{\nu j} B_{\nu k}-\frac{N-2}{4 N} B_{\nu j} B_{\mu k}\right. \\
& \left.-\frac{N+2}{4 N} B_{\mu j} B_{\nu k}\right) .
\end{aligned}
$$

The quantities $X_{j k l}^{\nu}$ and $Y_{j k l}^{\nu}$ are zero for elastic dumbbells.

Making use of these expressions we write various combinations in Eqs. (3.1)-(3.3) as follows for Rouse chains:

$$
\begin{aligned}
& \sum_{\mu} \phi_{\nu \mu}=\frac{1}{2} H \sum_{j}\left(\delta_{\nu-1, j}+\delta_{\nu j}\right) Q_{j}^{2}, \\
& \sum_{\mu} \phi_{\nu \mu} \mathbf{F}_{\nu}^{(\phi)}=-\frac{1}{2} H^{2} \sum_{j l}\left(\delta_{\nu-1, j}+\delta_{\nu j}\right) \bar{B}_{l \nu} Q_{j}^{2} \mathbf{Q}_{l}, \\
& \sum_{\mu} \mathbf{F}_{\nu \mu}^{(\phi)} \mathbf{R}_{\nu}=-H \sum_{j k} W_{j k}^{\nu} \mathbf{Q}_{j} \mathbf{Q}_{k}, \\
& \sum_{\mu} \mathbf{R}_{\nu}\left(\mathbf{F}_{\nu}^{(\phi)}+\mathbf{F}_{\mu}^{(\phi)}\right) \cdot \mathbf{F}_{\nu \mu}^{(\phi)}=H^{2} \sum_{j k l} X_{j k l}^{\nu}\left(\mathbf{Q}_{j} \cdot \mathbf{Q}_{k}\right) \mathbf{Q}_{l},
\end{aligned}
$$

$$
\sum_{\mu}\left(\mathbf{R}_{\nu}+\mathbf{R}_{\mu}\right) \mathbf{F}_{\nu \mu}^{(\phi)} \mathbf{R}_{\nu}=-H \sum_{j k l} Y_{j k l}^{v} \mathbf{Q}_{j} \mathbf{Q}_{k} \mathbf{Q}_{l},
$$

$$
\begin{aligned}
& \sum_{\mu} \mathbf{R}_{\nu} \mathbf{F}_{\nu \mu}^{(\phi)} \mathbf{R}_{\nu}=-H \sum_{j k l} Z_{j k l}^{\nu} \mathbf{Q}_{j} \mathbf{Q}_{k} Q_{l}, \\
& \frac{\partial}{\partial \mathbf{Q}_{k}} \cdot\left(\mathbf{F}_{\nu \mu}^{(\phi)} \mathbf{R}_{\nu}\right)=-H \sum_{i} U_{k i}^{\nu \mu} \mathbf{Q}_{i}, \\
& \frac{\partial}{\partial \mathbf{Q}_{k}} \cdot\left(\mathbf{F}_{\nu \mu}^{(\phi)} \mathbf{R}_{\nu} \mathbf{R}_{\nu}\right)=-H \sum_{i j} V_{k i j}^{\nu \mu} \mathbf{Q}_{i} \mathbf{Q}_{j} .
\end{aligned}
$$

Equations (A12) and (A13) are used to go from Eq. (3.2) to Eq. (4.6), and then Eqs. (A1)-(A6) and Eqs. (A14)-(A19) may be used to rewrite Eq. (3.3), thus 


$$
\begin{aligned}
& \mathbf{q}^{(\phi) \alpha}=\frac{H^{2}}{2 \zeta_{0}} \sum_{\nu j k l} X_{j k l}^{\nu} \iint_{0}^{1}\left(\mathbf{Q}_{j} \cdot \mathbf{Q}_{k}\right) \mathbf{Q}_{l} \Psi\left(\mathbf{r}-\xi \mathbf{R}_{\nu}, \mathbf{Q}^{N-1}, t\right) d \xi d \mathbf{Q}^{N-1}-\frac{H k T}{2 \zeta_{0}} \sum_{\nu \mu k i}\left(\bar{B}_{k \nu}+\bar{B}_{k \mu}\right) U_{k i}^{\nu \mu} \int \mathbf{Q}_{i} \Psi d \mathbf{Q}^{N-1} \\
& +\frac{1}{2} H \boldsymbol{\kappa}: \sum_{\nu j k l}\left(Z_{j k l}^{\nu}-Y_{j k l}^{\nu}\right) \int \mathbf{Q}_{j} \mathbf{Q}_{k} \mathbf{Q}_{l} \Psi d \mathbf{Q}^{N-1}+\frac{1}{12} H \boldsymbol{\kappa}:\left(\nabla \cdot \sum_{\nu i j k l} B_{\nu i}\left(3 Y_{j k l}^{\nu}-4 Z_{j k l}^{\nu}\right) \int \mathbf{Q}_{i} \mathbf{Q}_{j} \mathbf{Q}_{k} \mathbf{Q}_{l} \Psi d \mathbf{Q}^{N-1}\right) \\
& -\frac{H k T}{2 \zeta_{0}} \mathbf{a} \cdot \sum_{\nu \mu i k l}\left(\bar{B}_{k \nu} B_{\nu l}+\bar{B}_{k \mu} B_{\mu l}\right) U_{k i}^{\nu \mu} \int \mathbf{Q}_{l} \mathbf{Q}_{i} \Psi d \mathbf{Q}^{N-1}+\frac{H k T}{N \zeta_{0}} \mathbf{a} \cdot \sum_{\nu j k} W_{j k}^{\nu} \mathbf{Q}_{j} \mathbf{Q}_{k} \Psi d \mathbf{Q}^{N-1}+\frac{H k T}{4 \zeta_{0}} \mathbf{a} \cdot \sum_{\nu \mu i k}\left(\bar{B}_{k \nu}\right. \\
& \left.+\bar{B}_{k \mu}\right) V_{k i j}^{\nu \mu} \int \mathbf{Q}_{i} \mathbf{Q}_{j} \Psi d \mathbf{Q}^{N-1}+\frac{H k T}{N \zeta_{0}} \nabla \cdot \sum_{\nu j k} W_{j k}^{\nu} \int \mathbf{Q}_{j} \mathbf{Q}_{k} \Psi d \mathbf{Q}^{N-1}+\frac{H k T}{4 \zeta_{0}} \nabla \cdot \sum_{\nu \mu i j k}\left(\bar{B}_{k \nu}+\bar{B}_{k \mu}\right) V_{k i j}^{\nu \mu} \int \mathbf{Q}_{i} \mathbf{Q}_{j} \Psi d \mathbf{Q}^{N-1} .
\end{aligned}
$$

Use of Eqs. (A2) and (A7)-(A11) then allow us to combine some terms in Eq. (A20) to get Eq. (4.7)

A few words are in order in connection with developing Eq. (3.3). The expression for $\left[\left[\dot{\mathbf{r}}_{\nu}^{\alpha}-\mathbf{v}\right]\right]^{\alpha}$ needed in Eqs. (2.3) and (2.4) is

$$
\left[\left[\dot{\mathbf{r}}_{\nu}^{\alpha}-\mathbf{v}\right]\right]^{\alpha}=\left[\boldsymbol{\kappa} \cdot \mathbf{R}_{\nu}^{\alpha}\right]-\frac{1}{\zeta_{0} \Psi_{\alpha}} \frac{\partial}{\partial \mathbf{r}_{\nu}^{\alpha}}\left(k T_{\nu}^{\alpha} \Psi_{\alpha}\right)+\frac{1}{\zeta_{0}} \mathbf{F}_{\nu}^{(\phi) \alpha}
$$

The $\mathbf{F}_{\nu}^{(\phi) \alpha}$ term is substituted into Eq. (2.3) to give the first term in Eq. (3.3); the remaining terms are substituted into
Eq. (2.4) to give terms $2-9$ in Eq. (3.3), in which $\Psi_{\alpha}$ has the arguments $\mathbf{r}, \mathbf{Q}^{\alpha}, t$.

${ }^{1}$ R. B. Bird, C. F. Curtiss, R. C. Armstrong, and O. Hassager, Dynamics of Polymeric Liquids, Kinetic Theory, 2nd edition (Wiley-Interscience, New York, 1987), Vol. 2.

${ }^{2}$ B. H. A. A. van den Brule, "A contribution to the micro-rheological modeling of transport properties," Doctoral dissertation, Twente University, The Netherlands (1989); Rheol. Acta 28, 257 (1989); 29, 416 (1990).

${ }^{3}$ C. F. Curtiss and R. B. Bird, Adv. Polym. Sci. 125, 1 (1996); corrigenda: on p. 35, replace $\frac{1}{2}$ by $-\frac{1}{2}$ in the second line of Eq. (7.15); on p. 79, in Eq. (16.18), replace $\frac{1}{4}$ by $\frac{1}{4} \nabla$, and replace $\cdot(\nabla \mathbf{v})^{\dagger}$ by $:(\nabla \mathbf{v})^{\dagger}$.

${ }^{4}$ H. C. Öttinger and F. Petrillo, J. Rheol. 40, 857 (1996).

${ }^{5}$ R. B. Bird and C. F. Curtiss, Rheol. Acta 35, 103 (1996).

${ }^{6}$ R. B. Bird, C. F. Curtiss, and K. J. Beers, Rheol. Acta 36, 269 (1997).

${ }^{7}$ C. F. Curtiss and R. B. Bird, J. Chem. Phys. 106, 9899 (1997).

${ }^{8}$ J. H. Irving and J. G. Kirkwood, J. Chem. Phys. 18, 817 (1950).

${ }^{9}$ H. Grad, Commun. Pure Appl. Math. 2, 325 (1949). 